\title{
CLASSIFICATION OF TORIC MANIFOLDS OVER AN $n$-CUBE WITH ONE VERTEX CUT
}

\author{
SHO HASUI, HIDEYA KUWATA, MIKIYA MASUDA, AND SEONJEONG PARK
}

\begin{abstract}
We say that a complete nonsingular toric variety (called a toric manifold in this paper) is over $P$ if its quotient by the compact torus is homeomorphic to $P$ as a manifold with corners. Bott manifolds (or Bott towers) are toric manifolds over an $n$-cube $I^{n}$ and blowing them up at a fixed point produces toric manifolds over vc $\left(I^{n}\right)$ an $n$-cube with one vertex cut. They are all projective. On the other hand, Oda's 3-fold, the simplest non-projective toric manifold, is over $\mathrm{vc}\left(I^{3}\right)$. In this paper, we classify toric manifolds over $\mathrm{vc}\left(I^{n}\right)$ $(n \geq 3)$ as varieties and also as smooth manifolds. As a consequence, it turns out that (1) there are many non-projective toric manifolds over $\mathrm{vc}\left(I^{n}\right)$ but they are all diffeomorphic, and (2) toric manifolds over $\mathrm{vc}\left(I^{n}\right)$ in some class are determined by their cohomology rings as varieties among toric manifolds.
\end{abstract}

\section{INTRODUCTION}

A toric variety of complex dimension $n$ is a normal complex algebraic variety with an algebraic action of $\left(\mathbb{C}^{*}\right)^{n}$ having an open dense orbit. In this paper, we are concerned with complete nonsingular toric varieties and call them toric manifolds. As is well-known, the category of toric varieties is equivalent to the category of fans, by which the classification of toric manifolds as varieties reduces to a problem of combinatorics. Indeed, this fundamental fact enables us to classify toric manifolds of complex dimension $\leq 2$ as varieties and also as smooth manifolds (see [10], 15]).

However, the classification of fans up to isomorphism is not an easy task in general, and not much is known about the classification of toric manifolds as smooth (or topological) manifolds in complex dimension $\geq 3$. An intriguing problem posed in 13 for the classification of toric manifolds as smooth (or topological) manifolds is what is now called the cohomological rigidity problem, which asks whether toric manifolds are diffeomorphic (or homeomorphic) if their integral cohomology rings are isomorphic as graded rings.

Let $X$ be a toric manifold of complex dimension $n$. Its quotient by the compact torus $\left(S^{1}\right)^{n}$ of $\left(\mathbb{C}^{*}\right)^{n}$ is an $n$-dimensional manifold with corners. It is often homeomorphic to a simple polytope as a manifold with corners, e.g., this is the case when $X$ is projective or $n \leq 3$ but not the case in general (18). When the quotient is homeomorphic to $P$ as a manifold with corners, we say that $X$ is over $P$.

Date: July 29, 2018

2000 Mathematics Subject Classification. Primary 55N10, 57S15; Secondary 14M25.

Key words and phrases. toric manifold, polytope, Oda's 3-fold, moment-angle manifold, cohomological rigidity.

The third author was partially supported by JSPS Grant-in-Aid for Scientific Research $16 \mathrm{~K} 05152$. 
Toric manifolds over an $n$-cube $I^{n}$ are called Bott manifolds (or Bott towers) and form an interesting class of toric manifolds ([1]). A Bott tower is a sequence of an iterated $\mathbb{P}^{1}$-bundles starting with a point, where each $\mathbb{P}^{1}$-bundle is the projectivization of the Whitney sum of two complex line bundles, and a Bott manifold is the top manifold in the sequence. The cohomological rigidity problem is not solved even for Bott manifolds but many results have been produced in support of the affirmative answer to the problem, see [3, 4], [5], 6], 12].

Let $\mathrm{vc}\left(I^{n}\right)$ be an $n$-cube with one vertex cut. Blowing up Bott manifolds at a fixed point produces toric manifolds over vc $\left(I^{n}\right)$. They are all projective since so are Bott manifolds. On the other hand, Oda's 3-fold, which is known as the simplest non-projective toric manifold, is over vc $\left(I^{3}\right)$. So, it would be meaningful to classify toric manifolds over $\operatorname{vc}\left(I^{n}\right)$ as varieties and also as smooth manifolds, and we carry out the task in this paper.

In order to state our main results, we introduce some terminology. We assume $n \geq 3$. The boundary complex $\mathcal{C}_{\mathrm{n}}$ of the simplicial polytope dual to $\operatorname{vc}\left(I^{n}\right)$ is the underlying simplicial complex of the fans associated with the toric manifolds over $\operatorname{vc}\left(I^{n}\right)$. The simplicial complex $\mathcal{C}_{\mathrm{n}}$ has $2 n+1$ vertices, which we give labels $1,2, \ldots, 2 n+1$. The vertex corresponding to the facet of $\mathrm{vc}\left(I^{n}\right)$ obtained by cutting a vertex of an $n$-cube $I^{n}$ is a distinguished vertex and we label it $2 n+1$. There is a unique $(n-1)$-simplex in $\mathcal{C}_{\mathrm{n}}$ which does not intersect with the link of the vertex $2 n+1$. The $n$ vertices of the simplex are labelled by $1,2, \ldots, n$. Then the labels of the remaining vertices of $\mathcal{C}_{\mathrm{n}}$ are uniquely determined by requiring that the vertices $i$ and $n+i$ do not span a 1 -simplex of $\mathcal{C}_{\mathrm{n}}$ for each $i=1, \ldots, n$.

Let $X$ be a toric manifold over $\operatorname{vc}\left(I^{n}\right)$. We denote by $\mathbf{v}_{i}$ the primitive edge vector in the fan of $X$ corresponding to the vertex $i$ of $\mathcal{C}_{\mathrm{n}}$. Then $\left\{\mathbf{v}_{1}, \mathbf{v}_{2}, \ldots, \mathbf{v}_{n}\right\}$ forms a basis of the lattice of the fan so that we obtain an integer square matrix $A_{X}$ satisfying

$$
\left(\mathbf{v}_{n+1}, \mathbf{v}_{n+2}, \ldots, \mathbf{v}_{2 n}\right)=-\left(\mathbf{v}_{1}, \mathbf{v}_{2}, \ldots, \mathbf{v}_{n}\right) A_{X} .
$$

It is not difficult to see that $\operatorname{det} A_{X}=1$ if and only if $X$ is the blow-up of a Bott manifold at a fixed point, where the Bott manifold is associated with the fan obtained from the fan of $X$ by removing the vertex $2 n+1$. The following is our first main theorem, which follows from Propositions 5.5 5.6, 6.1, and 7.1.

Theorem 1.1. The determinant of $A_{X}$ above takes any integer when $X$ runs over all toric manifolds over $\mathrm{vc}\left(I^{n}\right)(n \geq 3)$ and is invariant under isomorphisms of the cohomology rings of the toric manifolds. Moreover, unless the determinant is one, the following three statements are equivalent for toric manifolds $X$ and $X^{\prime}$ over $\operatorname{vc}\left(I^{n}\right)$ :

(1) $X$ and $X^{\prime}$ are diffeomorphic,

(2) $H^{*}(X ; \mathbb{Z})$ and $H^{*}\left(X^{\prime} ; \mathbb{Z}\right)$ are isomorphic as graded rings,

(3) $\operatorname{det} A_{X}=\operatorname{det} A_{X^{\prime}}$.

In particular, there is only one diffeomorphism class for toric manifolds over $\mathrm{vc}\left(I^{n}\right)$ with determinant $q$ for each $q \neq 1$.

Remark. By the theorem above, the cohomological rigidity holds for toric manifolds over $\operatorname{vc}\left(I^{n}\right)$ with determinant $q \neq 1$. One can see that the cohomological rigidity holds for toric manifolds over $\mathrm{vc}\left(I^{n}\right)$ with determinant one if and only if it holds for Bott manifolds of complex dimension $n$. 
The classification of our toric manifolds as varieties is the following, which follows from Theorem 3.3, Propositions 8.1 and 8.3 .

Theorem 1.2. Let $\mathcal{V} \mathcal{A R}^{n}(q)$ denote the set of variety isomorphism classes of toric manifolds over $\mathrm{vc}\left(I^{n}\right)(n \geq 3)$ with determinant $q$. Then we have the following.

(1) $\mathcal{V} \mathcal{A R}^{n}(q)$ consists of a single element for each $q \neq 0,1,2$.

(2) $\mathcal{V} \mathcal{A R}^{n}(0)$ is parametrized by sequences $\left(b_{1}, \ldots, b_{n}\right)$ of integers with $\sum_{i=1}^{n} b_{i}=$ 1 up to cyclic permutation.

(3) If $X=X^{\prime}$ in $\mathcal{V} \mathcal{A R}^{n}(1)$, then the Bott manifolds corresponding to $X$ and $X^{\prime}$ are isomorphic as varieties.

(4) $\mathcal{V A R}^{n}(2)$ is parametrized by sequences of 1 and -1 of length $n$ with an odd number of 1 's up to cyclic permutation.

Moreover, all elements in $\mathcal{V} \mathcal{A R}^{n}(q)$ are projective when $q \neq 2$, and one element in $\mathcal{V} \mathcal{A R}^{n}(2)$ is projective while the others in $\mathcal{V} \mathcal{A R}^{n}(2)$ are non-projective.

Remark. Oda's 3-fold lies in $\mathcal{V} \mathcal{A R}^{3}(2)$. By (4) above, the cardinality of $\mathcal{V} \mathcal{A R}^{n}(2)$ is equal to the number of binary necklaces of length $n$ with an odd number of zeros, which has been studied in combinatorics. See [20, A000016]. Indeed, this number is known as

$$
\frac{1}{2 n} \sum_{\substack{d \mid n \\ d: \text { odd }}} \varphi(d) 2^{n / d}
$$

where $\varphi$ denotes Euler's totient function. It approaches infinity as $n$ approaches infinity.

Combining the two theorems above, we obtain two interesting corollaries.

(1) The toric manifold over $\mathrm{vc}\left(I^{n}\right)$ with determinant 2 as a smooth manifold admits a projective variety structure and a non-projective variety structure.

(2) We say that a toric manifold $X$ is cohomologically super-rigid if any toric manifold $Y$ such that $H^{*}(Y ; \mathbb{Z}) \cong H^{*}(X ; \mathbb{Z})$ as graded rings is isomorphic to $X$ as a variety. In general, it is not true that if $X$ is over $P$ and $H^{*}(Y ; \mathbb{Z}) \cong H^{*}(X ; \mathbb{Z})$ as graded rings, then $Y$ is also over $P$, but this is true when $P$ is $\operatorname{vc}\left(I^{n}\right)$, see [7, Section 6] 1 Therefore, it follows from the two theorems above that a toric manifold over $\operatorname{vc}\left(I^{n}\right)$ with determinant not equal to $0,1,2$ is cohomologically super-rigid.

This paper is organized as follows. We recall some basic facts about toric varieties in Section 2 In Section 3, we investigate the complete nonsingular fans associated with toric manifolds over vc $\left(I^{n}\right)$. We divide the family of the fans into four types and classify the fans up to isomorphism, which proves Theorem 1.2 except for the projectivity. We study general properties of the cohomology rings of our toric manifolds in Section 4 and then study isomorphism classes of the cohomology rings in each type in Sections 5. These observations lead to the invariance of our determinant under cohomology ring isomorphisms mentioned in Theorem 1.1. In Sections 6 and 7, we discuss smooth classification of our toric manifolds with determinant 0 and 2 respectively. We use the quotient construction of toric manifolds in the former case while we use moment-angle manifolds developed in toric topology in the latter case. Section 8 is devoted to the determination of (non-)projectivity of our toric manifolds. In Appendix, we give a proof to a key proposition used in Section 7 about moment-angle manifolds and finish with some remarks.

\footnotetext{
${ }^{1}$ In [7], they assumed that the orbit space of $Y$ is a simple polytope, but their proof is applicable when the orbit space of $Y$ is the dual of a simplicial sphere.
} 
Throughout this paper, all cohomology groups will be taken with integer coefficients unless otherwise stated.

\section{Preliminaries}

In this section, we prepare some notations and recall some basic facts about toric geometry. For more details we refer the reader to [2], [8], [10], [15].

Toric varieties and Fans. A toric variety is a normal variety $X$ that contains an algebraic torus $\left(\mathbb{C}^{*}\right)^{n}$ as a dense open subset, together with an action $\left(\mathbb{C}^{*}\right)^{n} \times X \rightarrow$ $X$ of $\left(\mathbb{C}^{*}\right)^{n}$ on $X$ that extends the natural action of $\left(\mathbb{C}^{*}\right)^{n}$ on itself. A complete nonsingular toric variety is called a toric manifold in this paper.

A fundamental result of toric geometry is that there is a bijection between toric varieties of complex dimension $n$ and rational fans of real dimension $n$, and toric manifolds correspond to complete nonsingular fans. More strongly, the category of toric varieties is equivalent to the category of fans.

Throughout this paper, toric varieties we consider are toric manifolds, so we focus on simplicial fans.

Let $\mathcal{K}$ be a simplicial complex with $m$ vertices. We identify the vertex set of $\mathcal{K}$ with the index set $[m]:=\{1, \ldots, m\}$. Consider a map

$$
\mathcal{V}:[m] \rightarrow \mathbb{Z}^{n} \text {. }
$$

Henceforth we will denote $\mathcal{V}(i)$ by $\mathbf{v}_{i}$. The pair $\Delta=(\mathcal{K}, \mathcal{V})$ is called a (simplicial) fan of dimension $n$ if it satisfies the following:

(1) $\mathbf{v}_{i}$ 's for $i \in I$ are linearly independent (over $\mathbb{R}$ ) whenever $I \in \mathcal{K}$

(2) $\operatorname{cone}(I) \cap \operatorname{cone}(J)=\operatorname{cone}(I \cap J)$ for $I, J \in \mathcal{K}$, where cone $(I)$ is the cone spanned by $\mathbf{v}_{i}$ 's for $i \in I$, that is,

$$
\operatorname{cone}(I)=\left\{\sum_{i \in I} \alpha_{i} \mathbf{v}_{i} \mid \alpha_{i} \geq 0 \text { for all } i \in I\right\} \text {. }
$$

The simplicial complex $\mathcal{K}$ is called the underlying simplicial complex of the fan $\Delta$, and we also say that $\Delta$ is over $\mathcal{K}$. The fan $\Delta$ is complete if $\bigcup_{I \in \mathcal{K}} \operatorname{cone}(I)=\mathbb{R}^{n}$ and nonsingular if the $\mathbf{v}_{i}$ 's for $i \in I$ form a part of a basis of $\mathbb{Z}^{n}$ whenever $I \in \mathcal{K}$. Two fans $\Delta$ and $\Delta^{\prime}$ are isomorphic if there is an isomorphism $\kappa: \mathcal{K} \rightarrow \mathcal{K}^{\prime}$ and $R \in \mathrm{GL}_{n}(\mathbb{Z})$ such that $\mathbf{v}_{\kappa(i)}^{\prime}=R \mathbf{v}_{i}$ for every $i \in[m]$. When the fan $\Delta$ is complete and nonsingular, we denote by $X(\Delta)$ the toric manifold associated with $\Delta$. We say that $X(\Delta)$ is over $P$ when the orbit space of $X(\Delta)$ by the compact subtorus of $\left(\mathbb{C}^{*}\right)^{n}$ is homeomorphic to $P$ as a manifold with corners.

Quotient construction of toric manifolds. Suppose that the fan $\Delta=(\mathcal{K}, \mathcal{V})$ is complete and nonsingular. We set

$$
Z:=\bigcup_{J \notin \mathcal{K}}\left\{\left(z_{1}, \ldots, z_{m}\right) \in \mathbb{C}^{m} \mid z_{j}=0 \text { for all } j \in J\right\}, \text { and } U(\mathcal{K}):=\mathbb{C}^{m} \backslash Z \text {. }
$$

The natural action of $\left(\mathbb{C}^{*}\right)^{m}$ on $\mathbb{C}^{m}$ leaves the subset $U(\mathcal{K})$ of $\mathbb{C}^{m}$ invariant. For $\mathbf{v}=\left[v_{1}, \ldots, v_{n}\right]^{T} \in \mathbb{Z}^{n}$, we define $\lambda_{\mathbf{v}}: \mathbb{C}^{*} \rightarrow\left(\mathbb{C}^{*}\right)^{n}$ by

$$
\lambda_{\mathbf{v}}(h):=\left(h^{v_{1}}, \ldots, h^{v_{n}}\right)
$$

and $\lambda_{\mathcal{V}}:\left(\mathbb{C}^{*}\right)^{m} \rightarrow\left(\mathbb{C}^{*}\right)^{n}$ by

$$
\lambda_{\mathcal{V}}\left(h_{1}, \ldots, h_{m}\right):=\lambda_{\mathbf{v}_{1}}\left(h_{1}\right) \cdots \lambda_{\mathbf{v}_{m}}\left(h_{m}\right)
$$


where $\mathbf{v}_{i}=\mathcal{V}(i) \in \mathbb{Z}^{n}$. The nonsingularity of the fan $\Delta$ implies that $\lambda_{\mathcal{V}}$ is surjective, so $\left(\mathbb{C}^{*}\right)^{m} / \operatorname{ker} \lambda_{\mathcal{V}}$ can be identified with $\left(\mathbb{C}^{*}\right)^{n}$ via $\lambda_{\mathcal{V}}$. Then

$$
X(\Delta):=U(\mathcal{K}) / \operatorname{ker} \lambda_{\mathcal{V}}
$$

has an induced effective action of $\left(\mathbb{C}^{*}\right)^{m} / \operatorname{ker} \lambda_{\mathcal{V}} \cong\left(\mathbb{C}^{*}\right)^{n}$ having an open dense orbit and finitely many orbits. Indeed, $X(\Delta)$ with this action of $\left(\mathbb{C}^{*}\right)^{n}$ is the toric manifold associated with the fan $\Delta$.

The cohomology ring of a toric manifold $X(\Delta)$. For each $i=1, \ldots, m$, the subset $D_{i} \subset X(\Delta)$ defined by $z_{i}=0$ is an invariant divisor fixed pointwise by the $\mathbb{C}^{*}$-subgroup $\lambda_{\mathbf{v}_{i}}\left(\mathbb{C}^{*}\right)$. The Poincaré dual of $D_{i}$ gives a cohomology class $\mu_{i}$ of degree 2 in the cohomology ring $H^{*}(X(\Delta))$.

Theorem 2.1 (Danilov-Jurkiewicz). The cohomology ring $H^{*}(X(\Delta))$ is isomorphic to $\mathbb{Z}\left[\mu_{1}, \ldots, \mu_{m}\right] / \mathcal{I}$ as a graded ring, where $\mathcal{I}$ is the ideal generated by the following two types of elements:

(1) $\prod_{i \in I} \mu_{i}(I \notin \mathcal{K})$, and

(2) $\sum_{i=1}^{m}\left\langle\mathbf{u}, \mathbf{v}_{i}\right\rangle \mu_{i}$ for any $\mathbf{u} \in \mathbb{Z}^{n}$,

where $\langle$,$\rangle denotes the standard scalar product on \mathbb{Z}^{n}$.

For a simplicial complex $\mathcal{K}$ on $[m]$, a subset $I \subset[m]$ is a minimal non-face of $\mathcal{K}$ if $I \notin \mathcal{K}$ but all proper subsets $I^{\prime}$ of $I$ are simplices of $\mathcal{K}$. It is known that every simplicial complex is uniquely determined by its minimal non-faces. Relation (1) in Theorem 2.1 is determined by the data of minimal non-faces of $\mathcal{K}$.

In this paper, we focus on toric manifolds over an $n$-cube with one vertex cut. Since they are closely related to toric manifolds over an $n$-cube, we shall briefly review them.

Bott manifolds and their cohomology rings (see 11, 12 for details). A toric manifold over an $n$-cube $I^{n}$ is known as a Bott manifold or a Bott tower. It can be obtained as the total space of an iterated $\mathbb{P}^{1}$-bundle starting with a point, where each $\mathbb{P}^{1}$-bundle is the projectivization of the Whitney sum of two complex line bundles. Let $\mathcal{B}_{n}$ be the simplicial complex on $[2 n]$ whose minimal non-faces are $\{i, n+i\}$ for $i=1, \ldots, n$. Note that $\mathcal{B}_{n}$ is isomorphic to the boundary complex of the simplicial polytope dual to $I^{n}$. The fan associated with a Bott manifold is of the form $\Delta=\left(\mathcal{B}_{n}, \mathcal{V}\right)$ such that the integer square matrix $A$ defined by

$$
\left(\mathbf{v}_{n+1}, \ldots, \mathbf{v}_{2 n}\right)=-\left(\mathbf{v}_{1}, \ldots, \mathbf{v}_{n}\right) A \quad\left(\mathbf{v}_{i}=\mathcal{V}(i)\right)
$$

is unipotent lower triangular. Since the fan $\Delta$ is determined by the matrix $A$, we may denote the Bott manifold $X(\Delta)$ by $X(A)$. Then, setting $x_{i}:=\mu_{n+i}$ for $i=1, \ldots, n$ in Theorem 2.1, one can see that

$$
H^{*}(X(A)) \cong \mathbb{Z}\left[x_{1}, \ldots, x_{n}\right] /\left(x_{i}\left(x_{i}+\sum_{j=1}^{i-1} a_{i j} x_{j}\right) \mid 1 \leq i \leq n\right)
$$

where $a_{i j}$ denotes the $(i, j)$-entry of $A$.

We note that if $\rho$ is an automorphism of $\mathcal{B}_{n}$, then $\left(\mathcal{B}_{n}, \mathcal{V} \circ \rho\right)$ defines the same fan as $\left(\mathcal{B}_{n}, \mathcal{V}\right)$ and the matrix $A$ will change into the matrix $A_{\rho}$ defined by

$$
\left(\mathbf{v}_{\rho(n+1)}, \ldots, \mathbf{v}_{\rho(2 n)}\right)=-\left(\mathbf{v}_{\rho(1)}, \ldots, \mathbf{v}_{\rho(n)}\right) A_{\rho} .
$$

The group $\operatorname{Aut}\left(\mathcal{B}_{n}\right)$ of automorphisms of $\mathcal{B}_{n}$ is generated by two types of elements. One is a permutation $\sigma$ on $[n]$. Indeed, since the minimal non-faces of $\mathcal{B}_{n}$ are 
$\{i, n+i\}$ for $i=1, \ldots, n, \sigma$ induces an automorphism of $\mathcal{B}_{n}$ by sending $n+i$ to $n+\sigma(i)$. The other is a permutation on $[2 n]$ which sends $i$ to either $i$ or $n+i$ for each $i=1, \ldots, n$.

\section{Classification of Fans}

Let $\mathcal{C}_{\mathrm{n}}$ be a simplicial complex on $[2 n+1](n \geq 2)$ whose minimal non-faces are $\{i, n+i\},\{i, 2 n+1\}(i=1, \ldots, n)$, and $\{n+1, \ldots, 2 n\}$. Note that $\mathcal{C}_{\mathrm{n}}$ is isomorphic to the boundary complex of the simplicial polytope dual to $\operatorname{vc}\left(I^{n}\right)$ an $n$-cube with one vertex cut, see Figure 1. Therefore, the fan associated with a toric manifold over $\operatorname{vc}\left(I^{n}\right)$ is over $\mathcal{C}_{\mathrm{n}}$. Conversely, it turns out that the toric manifold associated with a fan over $\mathcal{C}_{\mathrm{n}}$ is over vc $\left(I^{n}\right)$, see Remark 2 in Appendix.
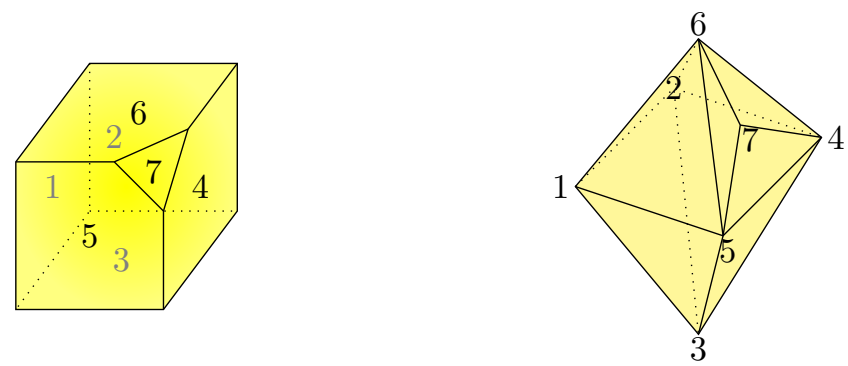

FiguRE 1. $\operatorname{vc}\left(I^{3}\right)$ and $\mathcal{C}_{3}=\partial\left(\operatorname{vc}\left(I^{3}\right)\right)^{*}$

Remark 1. When $n \geq 3$, the vertex of degree $n$ in $\mathcal{C}_{\mathrm{n}}$ is only $\{2 n+1\}$, and $\{1,2, \ldots, n\}$ is the unique maximal simplex of $\mathcal{C}_{\mathrm{n}}$ which does not intersect with the link of the vertex $\{2 n+1\}$. Therefore, any automorphism of $\mathcal{C}_{n}$ fixes $\{2 n+1\}$ and preserves $\{1, \ldots, n\}$. Since the minimal non-faces of $\mathcal{C}_{\mathrm{n}}$ are $\{i, n+i\}$ for $i=1, \ldots, n$, any automorphism of $\mathcal{C}_{\mathrm{n}}$ is induced from a permutation on [ $\left.n\right]$ by sending $n+i$ to $n+\sigma(i)$ when $n \geq 3$.

Let $\left(\mathcal{C}_{\mathrm{n}}, \mathcal{V}\right)$ be a complete nonsingular fan. Since $\{1, \ldots, n\}$ is a simplex of $\mathcal{C}_{\mathrm{n}}$, $\left\{\mathbf{v}_{1}, \ldots, \mathbf{v}_{n}\right\}$ is a basis of $\mathbb{Z}^{n}$. We express the remaining vectors $\mathbf{v}_{n+1}, \ldots, \mathbf{v}_{2 n}$, and $\mathbf{v}_{2 n+1}$ as linear combinations of the basis $\left\{\mathbf{v}_{1}, \ldots, \mathbf{v}_{n}\right\}$ as

$$
\left(\mathbf{v}_{n+1}, \ldots, \mathbf{v}_{2 n}\right)=-\left(\mathbf{v}_{1}, \ldots, \mathbf{v}_{n}\right) A \text {, and } \mathbf{v}_{2 n+1}=-\left(\mathbf{v}_{1}, \ldots, \mathbf{v}_{n}\right) \mathbf{b},
$$

where $A$ is an integer square matrix of size $n$ and $\mathbf{b}$ is an integer column vector of size $n$. The matrix $A$ and the vector $\mathbf{b}$ depend on a permutation $\sigma$ on $[n]$ as follows. Since $\left\{\mathbf{v}_{\sigma(1)}, \ldots, \mathbf{v}_{\sigma(n)}\right\}$ is a basis of $\mathbb{Z}^{n}$, an integer square matrix $A_{\sigma}$ and an integer column vector $\mathbf{b}_{\sigma}$ are defined by

$$
\begin{aligned}
\left(\mathbf{v}_{n+\sigma(1)}, \ldots, \mathbf{v}_{n+\sigma(n)}\right) & =-\left(\mathbf{v}_{\sigma(1)}, \ldots, \mathbf{v}_{\sigma(n)}\right) A_{\sigma}, \text { and } \\
\mathbf{v}_{2 n+1} & =-\left(\mathbf{v}_{\sigma(1)}, \ldots, \mathbf{v}_{\sigma(n)}\right) \mathbf{b}_{\sigma},
\end{aligned}
$$

respectively. Therefore, if $P_{\sigma}$ denotes the permutation matrix defined by

$$
\left(\mathbf{v}_{\sigma(1)}, \ldots, \mathbf{v}_{\sigma(n)}\right)=\left(\mathbf{v}_{1}, \ldots, \mathbf{v}_{n}\right) P_{\sigma},
$$

then

$$
\left(\mathbf{v}_{n+\sigma(1)}, \ldots, \mathbf{v}_{n+\sigma(n)}\right)=\left(\mathbf{v}_{n+1}, \ldots, \mathbf{v}_{2 n}\right) P_{\sigma}
$$


and plugging these into (3.2) and comparing the resulting equation with (3.1), we see that

$$
A_{\sigma}=P_{\sigma}^{-1} A P_{\sigma},
$$

in other words, the $(i, j)$-entry of $A_{\sigma}$ is equal to the $(\sigma(i), \sigma(j))$-entry of $A$. Combining (3.3) with Remark 1 shows that when $n \geq 3$, the fan $\Delta$ determines the matrix $A$ up to conjugation by permutation matrices, in particular, the value of $\operatorname{det} A$ is an invariant of the fan $\Delta$ when $n \geq 3$. One also notes that

$$
\text { the } i \text { th entry of } \mathbf{b}_{\sigma} \text { is equal to the } \sigma(i) \text { th entry of } \mathbf{b} \text {. }
$$

Motivated by this observation, we make the following definition.

Definition 3.1. A pair $\left(A^{\prime}, \mathbf{b}^{\prime}\right)$ is conjugate to $(A, \mathbf{b})$ if there is a permutation $\sigma$ on $[n]$ such that $\left(A^{\prime}, \mathbf{b}^{\prime}\right)=\left(A_{\sigma}, \mathbf{b}_{\sigma}\right)$.

For a subset $I$ of $[n]$, we define

$$
i(I)= \begin{cases}n+i & \text { if } i \in I, \\ i & \text { if } i \notin I .\end{cases}
$$

If $I \neq[n]$, then $\widetilde{I}:=\{1(I), \ldots, n(I)\}$ is a simplex of $\mathcal{C}_{\mathrm{n}}$, so $\left\{\mathbf{v}_{1(I)}, \ldots, \mathbf{v}_{n(I)}\right\}$ forms a basis of $\mathbb{Z}^{n}$. Therefore, the matrix $A_{I}$ defined by

$$
\left(\mathbf{v}_{1(I)}, \ldots, \mathbf{v}_{n(I)}\right)=-\left(\mathbf{v}_{1}, \ldots, \mathbf{v}_{n}\right) A_{I}
$$

is unimodular when $I$ is a proper subset of $[n]$. If $I \subsetneq J \subsetneq[n]$ and $J \backslash I$ consists of only one element, then cone $(\widetilde{I})$ and $\operatorname{cone}(\widetilde{J})$ are adjacent, i.e., their intersection cone $(\widetilde{I} \cap \widetilde{J})$ is a codimension one face of each. This implies that $\operatorname{det} A_{I}$ and $\operatorname{det} A_{J}$ have different signs. We note $A_{\emptyset}=-E_{n}$ where $E_{n}$ denotes the identity matrix of size $n$, and if $I$ consists of a single element $\{i\}$, then $A_{\{i\}}$ is $-E_{n}$ with the $i$ th column replaced by the $i$ th column of $A$. Since $\operatorname{det} A_{\emptyset}=(-1)^{n}$ and $\operatorname{det} A_{\{i\}}=(-1)^{n-1} a_{i i}$, where $a_{i i}$ is the $(i, i)$-entry of $A$, and they have different signs, we have $a_{i i}=1$. In general, $\operatorname{det} A_{I}$ is the principal minor of $A$ associated with $I$ multiplied by $(-1)^{n-|I|}$. Then an inductive argument on $|I|$ shows that all proper principal minors of $A$ are 1. This observation reminds us of the following lemma.

Lemma 3.1. 12, Lemma 3.3] Let $R$ be a commutative ring with unit 1 and $A$ be a square matrix of size $n$ with entries in $R$. Suppose that every proper principal minor of $A$ is equal to 1 . If $\operatorname{det} A=1$, then $A$ is conjugate by a permutation matrix to a unipotent lower triangular matrix, and otherwise to a matrix of the form

$$
\left[\begin{array}{ccccc}
1 & 0 & \ldots & 0 & a_{1} \\
a_{2} & 1 & \ldots & 0 & 0 \\
\vdots & \vdots & \ddots & \vdots & \vdots \\
0 & 0 & \ldots & 1 & 0 \\
0 & 0 & \ldots & a_{n} & 1
\end{array}\right]
$$

where $\operatorname{det} A=1+(-1)^{n-1} \prod_{i=1}^{n} a_{i}$ and all $a_{i}$ 's are nonzero since $\operatorname{det} A \neq 1$.

Applying Lemma 3.1 with $R=\mathbb{Z}$ to our matrix $A$, we may assume that $A$ is of the form in Lemma 3.1. Based on this understanding, we can characterize the pairs $(A, \mathbf{b})$ obtained from complete nonsingular fans over $\mathcal{C}_{\mathrm{n}}$. 
Proposition 3.2. Let $\mathcal{C}_{\mathrm{n}}$ be as above and $(A, \mathbf{b})$ be a pair which defines a fan over $\mathcal{C}_{\mathrm{n}}$ by (3.1). Then fans over $\mathcal{C}_{\mathrm{n}}$ are classified into four types according to the values of $\operatorname{det} A$. In the following, $\mathbf{a}_{i}$ denotes the $i$ th column vector of $A$ for $i=1, \ldots, n$.

Type $0(\operatorname{det} A=0)$

$$
\left[\begin{array}{ccccc}
1 & 0 & \ldots & 0 & -1 \\
-1 & 1 & \ldots & 0 & 0 \\
\vdots & \ddots & \ddots & \vdots & \vdots \\
0 & 0 & \ldots & 1 & 0 \\
0 & 0 & \ldots & -1 & 1
\end{array}\right], \quad \mathbf{b}=\left[\begin{array}{c}
b_{1} \\
b_{2} \\
\vdots \\
\vdots \\
b_{n}
\end{array}\right] \quad \text { with } \sum_{i=1}^{n} b_{i}=1
$$

Type $1(\operatorname{det} A=1)$

$$
\left[\begin{array}{ccccc}
1 & 0 & \cdots & 0 & 0 \\
a_{2,1} & 1 & \cdots & 0 & 0 \\
\vdots & \vdots & \ddots & \vdots & \vdots \\
a_{n-1,1} & a_{n-1,2} & \ldots & 1 & 0 \\
a_{n, 1} & a_{n, 2} & \ldots & a_{n, n-1} & 1
\end{array}\right], \quad \mathbf{b}=\sum_{i=1}^{n} \mathbf{a}_{i}
$$

Type $2(\operatorname{det} A=2)$

$$
\left[\begin{array}{ccccc}
1 & 0 & \ldots & 0 & a_{1} \\
a_{2} & 1 & \ldots & 0 & 0 \\
\vdots & \vdots & \ddots & \vdots & \vdots \\
0 & 0 & \ldots & 1 & 0 \\
0 & 0 & \ldots & a_{n} & 1
\end{array}\right], \quad \mathbf{b}=\frac{1}{2} \sum_{i=1}^{n} \mathbf{a}_{i}
$$

where $a_{i}= \pm 1$ for $i=1, \ldots, n$ and the number of $a_{i}$ 's equal to 1 is odd.

Type $3(\operatorname{det} A \neq 0,1,2)$

$$
\left[\begin{array}{ccccc}
1 & 0 & \ldots & 0 & a \\
-1 & 1 & \ldots & 0 & 0 \\
\vdots & \vdots & \ddots & \vdots & \vdots \\
0 & 0 & \ldots & 1 & 0 \\
0 & 0 & \ldots & -1 & 1
\end{array}\right] \quad a \neq 0, \pm 1, \quad \mathbf{b}=\frac{1}{\operatorname{det} A} \sum_{i=1}^{n} \mathbf{a}_{i}=\left[\begin{array}{c}
1 \\
0 \\
\vdots \\
\vdots \\
0
\end{array}\right]
$$

Remark. A fan of Type 1 is a blow-up of a fan of a Bott manifold explained in the last section. The matrix $A$ with $a=-1,0$, and 1 in Type 3 is of Type 0,1 , and 2, respectively.

Proof of Proposition 3.2. Since each $n$-dimensional cone containing $\mathbf{b}$ is nonsingular, the determinant of the matrix obtained from $A$ by replacing $\mathbf{a}_{i}$ with $\mathbf{b}$ is 1 up to sign but we can see that it is actually 1 by taking orientations into account, that is,

$$
\operatorname{det}\left[\mathbf{a}_{1}, \ldots, \mathbf{a}_{i-1}, \mathbf{b}, \mathbf{a}_{i+1}, \ldots, \mathbf{a}_{n}\right]=1, \quad \text { for } i=1,2, \ldots, n \text {. }
$$


Note that the equality above is equivalent to $\sum_{k=1}^{n} b_{k} A_{k i}=1$, where $A_{k i}$ is the $(k, i)$ minor of $A$ multiplied by $(-1)^{k+i}$. Hence, if we denote by $\tilde{A}$ the square matrix whose $(i, k)$-entry is $A_{k i}$, then we get

$$
\tilde{A} \mathbf{b}=\mathbf{1}
$$

where $\mathbf{1}=[1, \ldots, 1]^{T}$. Note that $A \tilde{A}=(\operatorname{det} A) E_{n}$, where $E_{n}$ is the identity matrix of size $n$.

Suppose that $\operatorname{det} A=0$. Then $A$ is of (3.4) and $A \tilde{A}$ is the zero matrix of size $n$, so if we denote by $\mathbf{r}_{i}$ the $i$ th row vector of $\tilde{A}$, then we have

$$
\mathbf{r}_{i}+a_{i} \mathbf{r}_{i-1}=0 \text { for } 1 \leq i \leq n,
$$

where $\mathbf{r}_{0}=\mathbf{r}_{n}$. Hence by adding $a_{i}$ times the $(i-1)$ th row to the $i$ th row in $[\tilde{A}, \mathbf{1}]$ from $i=n$ to $i=2$, we get

$$
\left[\begin{array}{ccccc}
1 & * & \ldots & * & 1 \\
0 & 0 & \ldots & 0 & 1+a_{2} \\
\vdots & \vdots & \ddots & \vdots & \vdots \\
0 & 0 & \ldots & 0 & 1+a_{n}
\end{array}\right]
$$

where $*$ 's are some integers. On the other hand, since $\mathbf{b}$ is a solution of $\tilde{A} \mathbf{x}=\mathbf{1}$, we have $\operatorname{rank}[\tilde{A}, \mathbf{1}]=\operatorname{rank} \tilde{A}$. Therefore $a_{2}=\cdots=a_{n}=-1$. Since $\operatorname{det} A=1+$ $(-1)^{n-1} \prod_{i=1}^{n} a_{i}=0$, we also have $a_{1}=-1$. Hence, $\operatorname{det}\left[\mathbf{a}_{1}, \ldots, \mathbf{a}_{n-1}, \mathbf{b}\right]=\sum_{i=1}^{n} b_{i}$ and $\sum_{i=1}^{n} b_{i}=1$ by 3.5 .

We now turn to the case $\operatorname{det} A \neq 0$. Since $A \tilde{A}=(\operatorname{det} A) E_{n}$, we obtain $\mathbf{b}=$ $\frac{1}{\operatorname{det} A} \sum_{i=1}^{n} \mathbf{a}_{i}$ from (3.6) regardless of the types. When $\operatorname{det} A=1$, this together with Lemma 3.1 establishes Type 1 case.

In Type 2, since $\operatorname{det} A=1+(-1)^{n-1} \prod_{i=1}^{n} a_{i}=2$ and $a_{i}$ 's are integers, we can see that $a_{i}= \pm 1$ for $i=1, \ldots, n$ and the number of $a_{i}$ 's equal to 1 is odd.

In Type 3 , we prove the following by using induction on $n$;

(*) The number of $a_{i}$ 's equal to -1 is $n-1$ if $\operatorname{det} A \neq 0,1,2$.

When $n=2$, we have

$$
\left[\mathbf{v}_{1}, \mathbf{v}_{2}, \mathbf{v}_{3}, \mathbf{v}_{5}, \mathbf{v}_{4}\right]=\left[-\mathbf{e}_{1},-\mathbf{e}_{2}, \mathbf{a}_{1}, \mathbf{b}, \mathbf{a}_{2}\right]=\left[\begin{array}{ccccc}
-1 & 0 & 1 & b_{1} & a_{1} \\
0 & -1 & a_{2} & b_{2} & 1
\end{array}\right]
$$

and

$$
\operatorname{det}\left[\mathbf{a}_{1}, \mathbf{b}\right]=b_{2}-a_{2} b_{1}=1 \text { and } \operatorname{det}\left[\mathbf{b}, \mathbf{a}_{2}\right]=b_{1}-a_{1} b_{2}=1
$$

from (3.5). This means that $\mathbf{v}_{1}, \mathbf{v}_{2}, \mathbf{v}_{3}, \mathbf{v}_{5}, \mathbf{v}_{4}$ are arranged in counterclockwise, and any consecutive two vectors in them form a basis of $\mathbb{Z}^{2}$. Therefore there exist integers $c_{1}, \ldots, c_{5}$ satisfying the equations

$$
\begin{aligned}
& \mathbf{v}_{1}+\mathbf{v}_{3}=c_{2} \mathbf{v}_{2}, \mathbf{v}_{2}+\mathbf{v}_{5}=c_{3} \mathbf{v}_{3}, \\
& \mathbf{v}_{3}+\mathbf{v}_{4}=c_{4} \mathbf{v}_{5}, \mathbf{v}_{5}+\mathbf{v}_{1}=c_{5} \mathbf{v}_{4}, \mathbf{v}_{4}+\mathbf{v}_{2}=c_{1} \mathbf{v}_{1},
\end{aligned}
$$

and an elementary computation shows that those integers are as follows:

$$
c_{1}=-a_{1}, c_{2}=-a_{2}, c_{3}=b_{1}, c_{4}=1-a_{1} a_{2}, c_{5}=b_{2} .
$$

The vectors $\mathbf{v}_{1}, \mathbf{v}_{2}, \mathbf{v}_{3}, \mathbf{v}_{5}, \mathbf{v}_{4}$ are primitive, arranged in counterclockwise, and form a 2-dimensional complete nonsingular fan, so the integers $c_{1}, \ldots, c_{5}$ must satisfy 
the equation 2

$$
\sum_{i=1}^{5} c_{i}=3 \times 5-12 .
$$

This together with (3.7) shows that $a_{1} a_{2}\left(a_{1}+1\right)\left(a_{2}+1\right)=0$. Combining this equality with the condition $\operatorname{det} A=1-a_{1} a_{2} \neq 0,1,2$, we see that exactly one of $a_{1}$ and $a_{2}$ is equal to -1 , and the other is different from $0, \pm 1$. Hence, statement $(*)$ holds for $n=2$.

Assume that statement $(*)$ holds for $n-1$ for $n \geq 3$. Let $\Delta_{1}$ be the fan obtained from $\Delta$ by projecting onto $\mathbb{Z}^{n} /\left\langle\mathbf{a}_{1}\right\rangle$. Then $\Delta_{1}$ is determined by the $(n-1) \times(n-1)$ matrix

$$
A_{1}=\left[\begin{array}{ccccc}
1 & 0 & \ldots & 0 & -a_{1} a_{2} \\
a_{3} & 1 & \ldots & 0 & 0 \\
\vdots & \vdots & \ddots & \vdots & \vdots \\
0 & 0 & \ldots & 1 & 0 \\
0 & 0 & \ldots & a_{n} & 1
\end{array}\right] .
$$

Since $\operatorname{det} A_{1}=\operatorname{det} A \neq 0,1,2$, it follows from the induction hypothesis that the number of $(-1)$ 's in $\left\{-a_{1} a_{2}, a_{3}, \ldots, a_{n}\right\}$ is equal to $n-2$. Repeating this procedure for $\mathbf{a}_{2}, \ldots, \mathbf{a}_{n}$, one sees that the number of $(-1)$ 's in

$$
\left\{a_{1}, \ldots, a_{i-1},-a_{i} a_{i+1}, a_{i+2}, \ldots, a_{n}\right\}
$$

is equal to $n-2$ for $i=1, \ldots, n$, where $a_{n+1}=a_{1}$. This implies statement $(*)$.

We note that any cyclic permutation on $[n]$ preserves the form of the matrix $A$ in (3.4) and permutes $a_{i}$ 's cyclically, so we may assume that the entry $a(\neq 0, \pm 1)$ in our matrix $A$ is placed in the $(1, n)$-entry through a cyclic permutation. This establishes Type 3 case.

Suppose that $n \geq 3$. Then a pair $(A, \mathbf{b})$ is associated with a complete nonsingular fan over $\mathcal{C}_{\mathrm{n}}$ through (3.1) but it is defined up to conjugation (see Definition 3.1) as observed before. By Proposition 3.2, we may assume that the pair $(A, \mathbf{b})$ is one of the form in the proposition. On the other hand, it is not difficult to see that the pair $(A, \mathbf{b})$ in Proposition 3.2 defines a complete nonsingular fan over $\mathcal{C}_{\mathrm{n}}$ through (3.1) up to isomorphism. However, it happens that two different pairs $(A, \mathbf{b})$ and $\left(A^{\prime}, \mathbf{b}^{\prime}\right)$ define isomorphic fans over $\mathcal{C}_{\mathrm{n}}$. For instance, as remarked at the end of the proof of Proposition [3.2 any cyclic permutation on $[n]$ preserves the form of the matrix $A$ in (3.4) and permutes $a_{i}$ 's cyclically.

The following theorem classifies complete nonsingular fans over $\mathcal{C}_{\mathrm{n}}$ up to isomorphism, in other words, toric manifolds over vc $\left(I^{n}\right)$ up to variety isomorphism, in terms of the pairs $(A, \mathbf{b})$.

Theorem 3.3. Suppose $n \geq 3$. Then pairs $(A, \mathbf{b})$ and $\left(A^{\prime}, \mathbf{b}^{\prime}\right)$ in Proposition 3.2 define isomorphic fans over $\mathcal{C}_{\mathrm{n}}$ if and only if they are of the same type in Proposition 3.2 and

(1) there is an integer $k \in[n]$ such that $b_{i}^{\prime}=b_{i+k}$ for every $i \in[n]$ in Type 0;

(2) there is a permutation matrix $P$ such that $A^{\prime}=P^{-1} A P$ in Type 1 ;

\footnotetext{
${ }^{2}$ If primitive integer vectors $\mathbf{w}_{1}, \mathbf{w}_{2}, \ldots, \mathbf{w}_{d}=\mathbf{w}_{0}$ in $\mathbb{Z}^{2}(d \geq 3)$ are arranged in counterclockwise and form a 2-dimensional complete nonsingular fan, then we must have $\mathbf{w}_{i-1}+\mathbf{w}_{i+1}=c_{i} \mathbf{w}_{i}$ with some integers $c_{i}$ for $1 \leq i \leq d$ and $\sum_{i=1}^{d} c_{i}=3 d-12$. See [10, pages 42-44].
} 
(3) there is an integer $k \in[n]$ such that $a_{i}^{\prime}=a_{i+k}$ for every $i \in[n]$ in Type 2; and

(4) $(A, \mathbf{b})=\left(A^{\prime}, \mathbf{b}^{\prime}\right)$ in Type 3 ,

where the indices in (1) and (3) above are taken modulo $n$.

Proof. As remarked before, $\{2 n+1\}$ is the unique vertex of $\mathcal{C}_{n}$ of degree $n$ since $n \geq 3$, and hence $\{1, \ldots, n\}$ is the unique maximal simplex of $\mathcal{C}_{\mathrm{n}}$ which does not intersect with the link of the vertex $\{2 n+1\}$. Therefore, the pair $(A, \mathbf{b})$ is associated with a fan $\Delta$ over $\mathcal{C}_{\mathrm{n}}$ up to conjugation by 3.1), and if two fans $\Delta$ and $\Delta^{\prime}$ over $\mathcal{C}_{\mathrm{n}}$ are isomorphic, then the induced isomorphism on $\mathcal{C}_{\mathrm{n}}$ must preserve the simplices $\{1, \ldots, n\},\{n+1, \ldots, 2 n\}$, and the vertex $\{2 n+1\}$. This implies that $\Delta$ and $\Delta^{\prime}$ are isomorphic if and only if the associated pairs $(A, \mathbf{b})$ and $\left(A^{\prime}, \mathbf{b}^{\prime}\right)$ are conjugate. In particular, the value of $\operatorname{det} A$ is invariant under isomorphisms of fans, which implies the first statement in the theorem.

If two pairs $(A, \mathbf{b})$ and $\left(A^{\prime}, \mathbf{b}^{\prime}\right)$ of Type 0 in Proposition 3.2 are conjugate, then there is a permutation $\sigma$ on $[n]$ such that $A^{\prime}=A_{\sigma}$ and $\mathbf{b}^{\prime}=\mathbf{b}_{\sigma}$ (see Definition 3.1). Here the $(i, j)$-entry of $A_{\sigma}$ is the $(\sigma(i), \sigma(j))$-entry of $A$. Since both $A^{\prime}=A_{\sigma}$ and $A$ are the matrix of Type $0, \sigma$ must be a cyclic permutation. Therefore (1) for Type 0 follows because the $i$ th entry of $\mathbf{b}_{\sigma}=\mathbf{b}^{\prime}$ is the $\sigma(i)$ th entry of $\mathbf{b}$.

In the other types, the vector $\mathbf{b}$ is determined by $A$. Since $A_{\sigma}$ is conjugate to $A$ by the permutation matrix associated with the permutation $\sigma$, (2) for Type 1 follows. As for Types 2 and 3, the permutation $\sigma$ must be a cyclic permutation similarly to Type 0 . This proves (3) for Type 2 . As for Type 3, the cyclic permutation $\sigma$ must be the identity, which proves (4) for Type 3 .

Remark. By (3) in Theorem 3.3, the number of isomorphism classes of fans of dimension $n$ in Type 2 is the number of ordered $n$ sets of 1 and -1 with an odd number of 1's up to cyclic permutation. The number is known in combinatorics (as mentioned in Introduction) as

$$
\frac{1}{2 n} \sum_{\substack{d \mid n \\ d: \text { odd }}} \varphi(d) 2^{n / d}
$$

where $\varphi$ denotes Euler's totient function. Since $\varphi(1)=1$, the number is greater than or equal to $2^{n-1} / n$, and the equality holds if and only if $n$ is a power of 2 .

\section{General properties of COHOmology Rings}

As observed in the previous section, a fan $\Delta=\left(\mathcal{C}_{\mathrm{n}}, \mathcal{V}\right)$ is determined by a pair $(A, \mathbf{b})$, so we shall denote the toric manifold $X(\Delta)$ by $X(A, \mathbf{b})$. In this section, we study general properties of the cohomology ring $H^{*}(X(A, \mathbf{b}))$.

We set $x_{i}:=\mu_{n+i}$ for $i=1, \ldots, n$ and $x:=\mu_{2 n+1}$ in Theorem 2.1, i.e., $x_{1}, \ldots, x_{n}, x$ correspond to $\mathbf{a}_{1}, \ldots, \mathbf{a}_{n}, \mathbf{b}$ respectively. Then one can see that

$$
H^{*}(X(A, \mathbf{b})) \cong \mathbb{Z}\left[x_{1}, \ldots, x_{n}, x\right] / \mathcal{I}(A, \mathbf{b}),
$$

where $\mathcal{I}(A, \mathbf{b})$ is the ideal generated by $\prod_{i=1}^{n} x_{i}$,

$$
x_{i}\left(\sum_{j=1}^{n} a_{i j} x_{j}+b_{i} x\right) \text {, and } x\left(\sum_{j=1}^{n} a_{i j} x_{j}+b_{i} x\right) \quad(i=1, \ldots, n),
$$


where $a_{i j}$ denotes the $(i, j)$-entry of $A$. Note that since $a_{i i}=1$, the former relation in (4.1) shows that $x_{i}^{2}$ can be expressed as a linear combination of $x_{i} x_{j}(1 \leq j \neq i \leq n)$ and $x x_{i}$ for $i=1, \ldots, n$.

Lemma 4.1. The elements $x_{1}, \ldots, x_{n}, x$ of $H^{2}(X(A, \mathbf{b}))$ satisfy the following.

(1) $x x_{1}=x x_{2}=\cdots=x x_{n}$, and

(2) $x^{2}=-(\operatorname{det} A) x x_{i}$.

Proof. In this proof, the indices of $x_{i}$ 's are taken modulo $n$ as usual. We take four cases according to the types of $A$.

In Type $0(\operatorname{det} A=0)$,

$$
a_{i j}= \begin{cases}1 & \text { if } i=j, \\ -1 & \text { if } j \equiv i-1 \quad(\bmod n), \quad \text { and } \quad \sum_{i=1}^{n} b_{i}=1 . \\ 0 & \text { otherwise }\end{cases}
$$

Hence, we have

$$
x\left(x_{i}-x_{i-1}+b_{i} x\right)=0 \text { for } i=1, \ldots, n .
$$

Summing up the equations in (4.2) over $i=1, \ldots, n$, we obtain $\left(\sum_{i=1}^{n} b_{i}\right) x^{2}=0$, and hence $x^{2}=0$ since $\sum_{i=1}^{n} b_{i}=1$. This proves (2). Plugging $x^{2}=0$ into (4.2), we obtain (1).

In Type $1(\operatorname{det} A=1)$, the matrix $A$ is a unipotent lower triangular matrix and the vector $\mathbf{b}$ is the sum of all the column vectors of $A$, so $b_{i}=1+\sum_{j=1}^{i-1} a_{i j}$ for $i=1, \ldots, n$. Hence, it follows from (4.1) that we have

$$
x\left(x_{i}+\sum_{j=1}^{i-1} a_{i j} x_{j}+\left(1+\sum_{j=1}^{i-1} a_{i j}\right) x\right)=0 \quad \text { for } i=1, \ldots, n .
$$

Rewriting these equations, we have

$$
\left(x x_{i}+x^{2}\right)+\sum_{j=1}^{i-1} a_{i j}\left(x x_{j}+x^{2}\right)=0 \text { for } i=1, \ldots, n .
$$

Taking $i=1$ above, we obtain $x^{2}+x x_{1}=0$. Then, taking $i=2$ above and using $x^{2}+x x_{1}=0$, we obtain $x^{2}+x x_{2}=0$. Repeating this argument, we see that $x^{2}+x x_{i}=0$ for any $i$, which proves both (1) and (2) for Type 1 .

In Type $2(\operatorname{det} A=2)$, we have

$$
a_{i j}= \begin{cases}1 & \text { if } i=j, \\ a_{i} & \text { if } j \equiv i-1 \quad(\bmod n), \quad \text { and } \quad b_{i}=\left(1+a_{i}\right) / 2, \\ 0 & \text { otherwise, }\end{cases}
$$

where $a_{i}= \pm 1$ for $i=1, \ldots, n$. We set $I=\left\{i \mid a_{i}=1\right\}$. The cardinality $|I|$ of $I$ is odd from Proposition 3.2. It follows from (4.1) and 4.3) that

$$
\begin{aligned}
x\left(x_{i-1}+x_{i}+x\right) & =0 \text { for } i \in I, \text { and } \\
x\left(-x_{i-1}+x_{i}\right) & =0 \text { for } i \notin I .
\end{aligned}
$$

Assume that $I=[n]$. Then (4.4) holds for any $i$. Subtracting $x\left(x_{i-1}+x_{i}+x\right)=0$ from $x\left(x_{i}+x_{i+1}+x\right)=0$, we get $x x_{i-1}=x x_{i+1}$ for any $i=1, \ldots, n$. This proves (1) since $n=|I|$ is odd. Then (4.4) implies $x^{2}=-2 x_{i} x$, which is (2). 
Now assume that $I=\left\{i_{1}, \ldots, i_{m}\right\} \neq[n]$, where $i_{1}<i_{2}<\cdots<i_{m}$. We divide the index set $[n]$ into $I_{1}, \ldots, I_{m}$ such that each $I_{k}=\left\{i_{k}, i_{k}+1, \ldots, i_{k+1}-1\right\}$ satisfies

$$
a_{i_{k}}=1, a_{i_{k}+1}=\cdots=a_{i_{k+1}-1}=-1 \quad \text { for } k=1, \ldots, m .
$$

From 4.5), we have $x x_{i-1}=x x_{i}$ for each $i \in I_{k} \backslash\left\{i_{k}\right\}$. Hence

$$
x x_{i_{k}}=x x_{i_{k}+1}=\cdots=x x_{i_{k+1}-1} \quad \text { for } k=1, \ldots, m .
$$

On the other hand, since $i_{k}, i_{k+1} \in I$, it follows from (4.4) that

$$
x^{2}=-x x_{i_{k}-1}-x x_{i_{k}}, \quad x^{2}=-x x_{i_{k+1}-1}-x x_{i_{k+1}} .
$$

Since $x x_{i_{k-1}}=x x_{i_{k}-1}$ and $x x_{i_{k}}=x x_{i_{k+1}-1}$ from (4.6), it follows from (4.7) that

$$
x x_{i_{k-1}}=x x_{i_{k+1}} \text { for } k=1, \ldots, m \text {. }
$$

Since $m$ is odd, this shows that $x x_{i_{k}}$ is independent of $k$ and hence $x^{2}=-2 x x_{i_{k}}$ by (4.6) and (4.7). This implies both (1) and (2).

In Type $3(\operatorname{det} A \neq 0,1,2)$, we have

$$
a_{i j}=\left\{\begin{array}{ll}
1 & \text { if } i=j, \\
a & \text { if }(i, j)=(1, n), \\
-1 & \text { if } j=i-1, \\
0 & \text { otherwise, }
\end{array} \quad \text { and } \quad \mathbf{b}=\left[\begin{array}{c}
1 \\
0 \\
\vdots \\
0
\end{array}\right] .\right.
$$

Hence, it follows from (4.1) that

$$
x\left(x_{1}+a x_{n}+x\right)=0 \quad \text { and } \quad x\left(-x_{i-1}+x_{i}\right)=0 \quad \text { for } i=2, \ldots, n .
$$

The latter equations above mean (1). Then the former equation above implies that $x^{2}=-(1+a) x x_{i}$ for any $i$, proving (2) since $\operatorname{det} A=1+a$.

Corollary 4.2. In the same situation as above, the following statements hold:

(1) $x^{n}$ is $(\operatorname{det} A)^{n-1}$ times a generator of $H^{2 n}(X(A, \mathbf{b}))$.

(2) $x_{i} x_{j}(1 \leq i<j \leq n)$ and $x x_{1}$ form an additive basis of $H^{4}(X(A, \mathbf{b}))$.

Proof. It follows from Lemma 4.1 that $x^{n}=(-\operatorname{det} A)^{n-1} x \prod_{i=1}^{n-1} x_{i}$. Here, since $\{2 n+1,1,2, \ldots, n-1\}$ is an $(n-1)$-simplex of $\mathcal{K}$ and our fan is nonsingular, $x \prod_{i=1}^{n-1} x_{i}$ is a generator of $H^{2 n}(X(A, \mathbf{b}))$. This proves (1).

It follows from (4.1) and Lemma4.1 that $x_{i} x_{j}(1 \leq i<j \leq n)$ and $x x_{1}$ generate $H^{4}(X(A, \mathbf{b}))$. On the other hand, the rank of $H^{4}(X(A, \mathbf{b}))$ is $\left(\begin{array}{l}n \\ 2\end{array}\right)+1$. This can be seen by computing the $h$-vector of the simplicial complex $\mathcal{C}_{\mathrm{n}}$. Another way to see this fact is that the connected sum of $\left(\mathbb{C} P^{1}\right)^{n}$ and $\mathbb{C} P^{n}$ with reversed orientation is a toric manifold over vc $\left(I^{n}\right)$ and it has the same Betti numbers as $X(A, \mathbf{b})$. Since $\left(\begin{array}{l}n \\ 2\end{array}\right)+1$ is the number of the generators $x_{i} x_{j}(1 \leq i<j \leq n)$ and $x x_{1}$, they must be an additive basis of $H^{4}(X(A, \mathbf{b}))$.

For an element $z$ of $H^{2}(X(A, \mathbf{b}))$, we define

$$
\operatorname{Ann}(z)=\left\{w \in H^{2}(X(A, \mathbf{b})) \mid z w=0 \text { in } H^{*}(X(A, \mathbf{b}))\right\} .
$$

Since $\{i, 2 n+1\}$ for $i=1, \ldots, n$ is a non-face of $\mathcal{C}_{\mathrm{n}}$, we have $x x_{i}=0$ and hence $\operatorname{Ann}(c x)$ is of rank $n$ for a nonzero constant $c$. The following lemma shows that the converse is also true. 
Lemma 4.3. For $n \geq 3$, if $\operatorname{Ann}(z)$ is of rank $n$, then $z$ is a nonzero constant multiple of $x$

Proof. Set $z=\sum_{i=1}^{n} c_{i} x_{i}+c x$. We will show that $c_{i}=0$ for $i=1, \ldots, n$ when $\operatorname{Ann}(z)$ is of rank $n$. If an element $\sum_{i=1}^{n} d_{i} x_{i}+d x$ belongs to $\operatorname{Ann}(z)$, then we have

$$
\left(\sum_{i=1}^{n} c_{i} x_{i}+c x\right)\left(\sum_{i=1}^{n} d_{i} x_{i}+d x\right)=0 .
$$

In the following we will use the fact that $x_{i} x_{j}(1 \leq i<j \leq n)$ and $x x_{1}$ form an additive basis of $H^{4}(X(A, \mathbf{b}))$, see Corollary $4.2(2)$.

In Type 1 , the matrix $A$ is a unipotent lower triangular matrix and we have the relations $x_{i}^{2}=-\sum_{j=1}^{i-1} a_{i j} x_{j} x_{i}-b_{i} x_{i} x(1 \leq i \leq n)$ from 4.1). Plugging this into (4.8), we can see that the coefficient of $x_{i} x_{j}(i>j)$ is $c_{i} d_{j}+c_{j} d_{i}-a_{i j} c_{i} d_{i}$ and the coefficients of $x_{2} x_{1}, x_{3} x_{2}$ and $x_{3} x_{1}$ satisfy

$$
\left[\begin{array}{ccc}
c_{2} & c_{1}-c_{2} a_{21} & 0 \\
0 & c_{3} & c_{2}-c_{3} a_{32} \\
c_{3} & 0 & c_{1}-c_{3} a_{31}
\end{array}\right]\left[\begin{array}{l}
d_{1} \\
d_{2} \\
d_{3}
\end{array}\right]=\left[\begin{array}{l}
0 \\
0 \\
0
\end{array}\right] .
$$

Since $\operatorname{Ann}(z)$ is of rank $n$, the leftmost matrix above is of rank at most one. Hence, we get $c_{1}=c_{2}=c_{3}=0$. The coefficients of $x_{i} x_{1}$ and $x_{i} x_{2}$ for $3 \leq i \leq n$ satisfy

$$
\left[\begin{array}{ccc}
c_{i} & 0 & c_{1}-c_{i} a_{i 1} \\
0 & c_{i} & c_{2}-c_{i} a_{i 2}
\end{array}\right]\left[\begin{array}{l}
d_{1} \\
d_{2} \\
d_{i}
\end{array}\right]=\left[\begin{array}{l}
0 \\
0
\end{array}\right] \text { for } 3 \leq i \leq n .
$$

Therefore $c_{i}=0$ for $3 \leq i \leq n$ by the same reason as above, proving the lemma for Type 1.

In the other types, the matrix $A$ is of the form (3.4), and we have relations

$$
x_{i}^{2}=-a_{i} x_{i} x_{i-1}-b_{i} x_{i} x \quad \text { for } 1 \leq i \leq n,
$$

from (4.1). Plugging these into (4.8), we can see that the coefficient of $x_{i} x_{i-1}$ is $c_{i-1} d_{i}-a_{i} c_{i} d_{i}+c_{i} d_{i-1}$ for $1 \leq i \leq n$ and hence we get

$$
\left[\begin{array}{ccccc}
c_{n}-c_{1} a_{1} & 0 & \ldots & 0 & c_{1} \\
c_{2} & c_{1}-c_{2} a_{2} & \ldots & 0 & 0 \\
\vdots & \vdots & \ddots & \vdots & \vdots \\
0 & 0 & \ldots & c_{n-2}-c_{n-1} a_{n-1} & 0 \\
0 & 0 & \ldots & c_{n} & c_{n-1}-c_{n} a_{n}
\end{array}\right]\left[\begin{array}{c}
d_{1} \\
d_{2} \\
\vdots \\
d_{n-1} \\
d_{n}
\end{array}\right]=\left[\begin{array}{c}
0 \\
0 \\
\vdots \\
0 \\
0
\end{array}\right]
$$

Since $\operatorname{Ann}(z)$ is of rank $n$, the rank of the leftmost matrix above is at most one. Hence, we get $c_{i}=0$ for $1 \leq i \leq n$.

\section{ISOMORPHISM CLASSES OF COHOMOLOGY RINGS IN EACH TYPE}

In this section we study isomorphism classes of the cohomology rings $H^{*}(X(A, \mathbf{b}))$ in each type. It turns out that the value of $\operatorname{det} A$ is preserved under isomorphisms of the cohomology rings and there is only one isomorphism class for each value of $\operatorname{det} A$ unless $\operatorname{det} A=1$ (Proposition [5.6). 
5.1. Type 0. The cohomology ring of $X(A, \mathbf{b})$ in Type 0 is

$$
H^{*}(X(A, \mathbf{b})) \cong \mathbb{Z}\left[x_{1}, \ldots, x_{n}, x\right] / \mathcal{I}(A, \mathbf{b}),
$$

where $\mathcal{I}(A, \mathbf{b})$ is the ideal generated by homogeneous polynomials

(1) $\prod_{j=1}^{n} x_{j}$ and

(2) $x_{i}\left(-x_{i-1}+x_{i}+b_{i} x\right)$ and $x\left(-x_{i-1}+x_{i}+b_{i} x\right)$ for $1 \leq i \leq n$,

where $x_{0}=x_{n}$.

Lemma 5.1. Cohomology rings of toric manifolds of Type 0 are isomorphic to each other.

Proof. Given $\mathbf{b}=\left[b_{1}, \ldots, b_{n}\right]^{T}$, let $\ell$ be the integer satisfying $\sum_{i=1}^{n-1}(n-i) b_{i} \equiv \ell$ $(\bmod n)$ and $0 \leq \ell \leq n-1$. Let $\mathbf{b}^{\prime}=\left[b_{1}^{\prime}, \ldots, b_{n}^{\prime}\right]^{T}$ such that

$$
b_{n-\ell}^{\prime}=1 \quad \text { and } \quad b_{i}^{\prime}=0 \text { for } i \neq n-\ell .
$$

Then $\sum_{i=1}^{n-1}(n-i) b_{i}^{\prime}=\ell$. Note that $\left(A, \mathbf{b}^{\prime}\right)$ is conjugate to $\left(A, \mathbf{b}_{\sigma}^{\prime}\right)$ for any cyclic permutation $\sigma$ on $[n]$ since $A_{\sigma}=A$ in Type 0 (see Definition 3.1 for the conjugation). Hence, it suffices to show that $H^{*}(X(A, \mathbf{b}))$ is isomorphic to $H^{*}\left(X\left(A, \mathbf{b}^{\prime}\right)\right)$.

Since $\sum_{i=1}^{n-1}(n-i) b_{i} \equiv \ell \equiv \sum_{i=1}^{n-1}(n-i) b_{i}^{\prime}(\bmod n)$, there exists an integer $\alpha$ such that

$$
\sum_{i=1}^{n-1}(n-i) b_{i}-\sum_{i=1}^{n-1}(n-i) b_{i}^{\prime}+n \alpha=0 .
$$

Then the integers $c_{i}$ 's defined by

$$
c_{i}=\sum_{j=1}^{i}\left(b_{j}-b_{j}^{\prime}\right)+\alpha \text { for } 1 \leq i \leq n-1 \quad \text { and } \quad c_{n}=\alpha
$$

satisfy that

$$
\sum_{i=1}^{n} c_{i}=0 \quad \text { and } \quad c_{i}-c_{i-1}=b_{i}-b_{i}^{\prime} \text { for } 1 \leq i \leq n,
$$

where $c_{0}=c_{n}$ (note that $\sum_{i=1}^{n} b_{i}=\sum_{i=1}^{n} b_{i}^{\prime}=1$ ).

We now consider the automorphism $\varphi$ of $\mathbb{Z}\left[x_{1}, \ldots, x_{n}, x\right]$ defined by

$$
\varphi\left(x_{i}\right)=x_{i}+c_{i} x \quad \text { for } 1 \leq i \leq n \text { and } \quad \varphi(x)=x .
$$

We shall check that $\varphi$ induces an isomorphism from $H^{*}\left(X\left(A, \mathbf{b}^{\prime}\right)\right)$ to $H^{*}(X(A, \mathbf{b}))$. First we have

$$
\begin{aligned}
\varphi\left(x_{i}\left(-x_{i-1}+x_{i}+b_{i}^{\prime} x\right)\right) & =\left(x_{i}+c_{i} x\right)\left(-x_{i-1}+x_{i}+\left(-c_{i-1}+c_{i}+b_{i}^{\prime}\right) x\right) \\
& \left.=\left(x_{i}+c_{i} x\right)\left(-x_{i-1}+x_{i}+b_{i} x\right) \quad \text { (by (5.1) }\right) \\
& =0 \quad \text { in } H^{*}(X(A, \mathbf{b})) .
\end{aligned}
$$

Similarly we have

$$
\varphi\left(x\left(-x_{i-1}+x_{i}+b_{i}^{\prime} x\right)\right)=x\left(-x_{i-1}+x_{i}+b_{i} x\right)=0 \quad \text { in } H^{*}(X(A, \mathbf{b})) .
$$


Finally we have

$$
\begin{array}{rlrl}
\varphi\left(\prod_{i=1}^{n} x_{i}\right) & =\prod_{i=1}^{n}\left(x_{i}+c_{i} x\right) & & \\
& =\sum_{i=1}^{n}\left(c_{i} x \prod_{j \neq i}^{n} x_{j}\right) & & \left(\because \prod_{i=1}^{n} x_{i}=0 \text { and } x^{2}=0\right. \text { by Lemma 4.1) } \\
& =\left(\sum_{i=1}^{n} c_{i}\right) x x_{1}^{n-1} & \left(\because x x_{1}=x x_{i} \text { for any } i\right. \text { by Lemma 4.1) } \\
& =0 & \left(\because \sum_{i=1}^{n} c_{i}=0 \text { by (5.1) }\right)
\end{array}
$$

in $H^{*}(X(A, \mathbf{b}))$. This proves that $\varphi$ induces a graded ring homomorphism $\hat{\varphi}$ from $H^{*}\left(X\left(A, \mathbf{b}^{\prime}\right)\right)$ to $H^{*}(X(A, \mathbf{b}))$. Similarly, the inverse of $\varphi$ induces a graded ring homomorphism in the opposite direction and gives the inverse of $\hat{\varphi}$, proving the lemma.

5.2. Type 1. Recall that a toric manifold $X(A, \mathbf{b})$ of Type 1 is a blow up of a Bott manifold $X(A)$ at a torus fixed point. We have

$$
H^{*}(X(A)) \cong \mathbb{Z}\left[x_{1}, \ldots, x_{n}\right] / \mathcal{I}(A)
$$

where $\mathcal{I}(A)$ is the ideal generated by

$$
x_{i}\left(x_{i}+\sum_{j=1}^{i-1} a_{i j} x_{j}\right) \quad(1 \leq i \leq n)
$$

by (2.1). On the other hand, as observed in Section 4 we have

$$
H^{*}(X(A, \mathbf{b})) \cong \mathbb{Z}\left[x_{1}, \ldots, x_{n}, x\right] / \mathcal{I}(A, \mathbf{b}),
$$

where $\mathcal{I}(A, \mathbf{b})$ is the ideal generated by homogeneous polynomials

(1) $\prod_{i=j}^{n} x_{j}$ and

(2) $x_{i}\left(x_{i}+\sum_{j=1}^{i-1} a_{i j} x_{j}+b_{i} x\right), x\left(x_{i}+\sum_{j=1}^{i-1} a_{i j} x_{j}+b_{i} x\right)$ for $1 \leq i \leq n$,

where $b_{i}=1+\sum_{j=1}^{i-1} a_{i j}$.

Lemma 5.2. Suppose $n \geq 3$. Then $H^{*}(X(A, \mathbf{b}))$ and $H^{*}\left(X\left(A^{\prime}, \mathbf{b}^{\prime}\right)\right)$ in Type 1 are isomorphic as graded rings if and only if $H^{*}(X(A))$ and $H^{*}\left(X\left(A^{\prime}\right)\right)$ are isomorphic as graded rings.

Proof. Since $X(A, \mathbf{b})$ is a blow up of $X(A)$ at a point, $X(A, \mathbf{b})$ is diffeomorphic to the connected sum of $X(A)$ and $\mathbb{C} P^{n}$ with reversed orientation. Therefore, the "if" part is obvious.

Let $\hat{\varphi}: H^{*}(X(A, \mathbf{b})) \rightarrow H^{*}\left(X\left(A^{\prime}, \mathbf{b}^{\prime}\right)\right)$ be a graded ring isomorphism. Since $\left\{x_{1}, \ldots, x_{n}, x\right\}$ is an additive basis of $H^{2}(X(A, \mathbf{b})), \hat{\varphi}$ induces an automorphism $\varphi$ of $\mathbb{Z}\left[x_{1}, \ldots, x_{n}, x\right]$ such that $\varphi(\mathcal{I}(A, \mathbf{b}))=\mathcal{I}\left(A^{\prime}, \mathbf{b}^{\prime}\right)$. By Lemma 4.3. $\varphi(x)=x$ up to sign, so $\varphi$ induces an automorphism $\bar{\varphi}$ of $\mathbb{Z}\left[x_{1}, \ldots, x_{n}\right]$ such that $\bar{\varphi}(\mathcal{I}(A))=\mathcal{I}\left(A^{\prime}\right)$. This proves the "only if" part of the lemma.

It is conjectured that if two Bott manifolds $X(A)$ and $X\left(A^{\prime}\right)$ have isomorphic cohomology rings, then they are diffeomorphic, and this conjecture is affirmatively solved when 
(1) the complex dimension of $X(A)$ is at most four, or

(2) $H^{*}(X(A) ; \mathbb{F})$ is isomorphic to $H^{*}\left(\left(\mathbb{P}^{1}\right)^{n} ; \mathbb{F}\right)$ for $\mathbb{F}=\mathbb{Q}$ or $\mathbb{Z} / 2 \mathbb{Z}$, see [3], 4], [5], 6]. Combining these results with Lemma 5.2, we get the following.

Corollary 5.3. Two toric manifolds $X(A, \mathbf{b})$ and $X\left(A^{\prime}, \mathbf{b}^{\prime}\right)$ in Type 1 are diffeomorphic if their cohomology rings are isomorphic as graded rings and the corresponding Bott manifolds $X(A)$ and $X\left(A^{\prime}\right)$ satisfy one of the conditions above.

5.3. Type 2. The cohomology ring of $X(A, \mathbf{b})$ in Type 2 is

$$
H^{*}(X(A, \mathbf{b})) \cong \mathbb{Z}\left[x_{1}, \ldots, x_{n}, x\right] / \mathcal{I}(A, \mathbf{b}),
$$

where $\mathcal{I}(A, \mathbf{b})$ is the ideal generated by homogeneous polynomials

(1) $x_{i}\left(x_{i}+a_{i} x_{i-1}+b_{i} x\right), x\left(x_{i}+a_{i} x_{i-1}+b_{i} x\right)$ for $1 \leq i \leq n$, and

(2) $\prod_{i=1}^{n} x_{i}$

where $b_{i}=\left(1+a_{i}\right) / 2$.

Lemma 5.4. Cohomology rings of toric manifolds of Type 2 are isomorphic to each other.

Proof. The matrix $A$ in Type 2 is determined by its entries $\left\{a_{1}, \ldots, a_{n}\right\}$. Assume that the number of $a_{i}$ 's equal to -1 , denoted by $m_{A}$, is greater than one. Since $n-$ $m_{A}$ is odd, it suffices to show that $H^{*}(X(A, \mathbf{b}))$ and $H^{*}\left(X\left(A^{\prime}, \mathbf{b}^{\prime}\right)\right)$ are isomorphic as graded rings whenever $m_{A}-m_{A^{\prime}}=2$.

In the following, the indices of $a_{i}$ 's are taken modulo $n$ as usual. Since the number of $a_{i}$ 's equal to 1 is odd, there exist $i<j$ such that

$$
a_{i}=-1, a_{i+1}=\cdots=a_{j-1}=1, a_{j}=-1, \quad \text { and } j-i \text { is even. }
$$

Then we have

$$
x_{k}+a_{k} x_{k-1}+b_{k} x= \begin{cases}x_{k}-x_{k-1} & \text { for } k=i \text { or } j, \\ x_{k}+x_{k-1}+x & \text { for } i<k<j .\end{cases}
$$

We set

$$
a_{k}^{\prime}= \begin{cases}1 & \text { for } i \leq k \leq j \\ a_{k} & \text { otherwise }\end{cases}
$$

Consider the automorphism $\varphi$ of $\mathbb{Z}\left[x_{1}, \ldots, x_{n}, x\right]$ defined by

$$
\varphi\left(x_{k}\right)=\left\{\begin{array}{ll}
-\left(x_{k}+x\right) & \text { for } i \leq k \leq j-1, \\
x_{k} & \text { otherwise, }
\end{array} \text { and } \quad \varphi(x)=x .\right.
$$

We will show that $\varphi$ induces a graded ring isomorphism from $H^{*}\left(X\left(A^{\prime}, \mathbf{b}^{\prime}\right)\right)$ to $H^{*}(X(A, \mathbf{b}))$. One can easily check that

$$
\varphi\left(x_{k}+a_{k}^{\prime} x_{k-1}+b_{k}^{\prime} x\right)= \begin{cases}-\left(x_{k}+a_{k} x_{k-1}+b_{k} x\right) & (i \leq k \leq j-1), \\ x_{k}+a_{k} x_{k-1}+b_{k} x & \text { otherwise. }\end{cases}
$$

This together with (5.2) shows that both $\varphi\left(x_{k}\left(x_{k}+a_{k}^{\prime} x_{k-1}+b_{k}^{\prime} x\right)\right)$ and $\varphi\left(x\left(x_{k}+\right.\right.$ $\left.\left.a_{k}^{\prime} x_{k-1}+b_{k}^{\prime} x\right)\right)$ are in $\mathcal{I}(A, \mathbf{b})$ for $k=1, \ldots, n$.

Let us check that $\varphi\left(\prod_{k=1}^{n} x_{k}\right)=\prod_{k=1}^{n} x_{k}$ in $H^{*}(X(A, \mathbf{b}))$. Since $j-i$ is even, it follows from (5.2) that

$$
\varphi\left(\prod_{k=1}^{n} x_{k}\right)=\prod_{k=i}^{j-1}\left(x_{k}+x\right) \prod_{\text {otherwise }} x_{k} .
$$


Recall that $x^{2}=-2 x x_{k}$ and $x x_{1}=x x_{k}$ for any $k$ by Lemma 4.1. Then in $H^{*}(X(A, \mathbf{b}))$ we have

$$
\begin{aligned}
& \prod_{k=i}^{j-1}\left(x_{k}+x\right) \\
= & \prod_{k=i}^{j-1} x_{k}+\left(\left(\begin{array}{c}
j-i \\
1
\end{array}\right)+\left(\begin{array}{c}
j-i \\
2
\end{array}\right)(-2)+\cdots+\left(\begin{array}{c}
j-i \\
j-i
\end{array}\right)(-2)^{j-i-1}\right) x x_{1}^{j-i-1} \\
= & \prod_{k=i}^{j-1} x_{k}-\frac{1}{2}\left((1+(-2))^{j-i}-1\right) x x_{1}^{j-i-1} \\
= & \prod_{k=i}^{j-1} x_{k} \quad(\because j-i \text { is even }) .
\end{aligned}
$$

This together with (5.3) shows that $\varphi\left(\prod_{k=1}^{n} x_{k}\right)=\prod_{k=1}^{n} x_{k}$ in $H^{*}(X(A, \mathbf{b}))$.

Thus, $\varphi$ induces a graded ring homomorphism $\hat{\varphi}: H^{*}\left(X\left(A^{\prime}, \mathbf{b}^{\prime}\right)\right) \rightarrow H^{*}(X(A, \mathbf{b}))$. Similarly, the inverse of $\varphi$ induces a graded ring homomorphism in the opposite direction and gives the inverse of $\hat{\varphi}$, proving the lemma.

5.4. Type 3. The cohomology ring of $X(A, \mathbf{b})$ in Type 3 is

$$
H^{*}(X(A, \mathbf{b})) \cong \mathbb{Z}\left[x_{1}, \ldots, x_{n}, x\right] / \mathcal{I}(A, \mathbf{b}),
$$

where $\mathcal{I}(A, \mathbf{b})$ is the ideal generated by homogeneous polynomials

(1) $\prod_{j=1}^{n} x_{j}, x_{1}\left(x_{1}+a x_{n}+x\right), x\left(x_{1}+a x_{n}+x\right)$ and

(2) $x_{i}\left(x_{i-1}-x_{i}\right), x\left(x_{i-1}-x_{i}\right)$ for $2 \leq i \leq n$.

Proposition 5.5. When $n \geq 3, H^{*}(X(A, \mathbf{b}))$ and $H^{*}\left(X\left(A^{\prime}, \mathbf{b}^{\prime}\right)\right)$ in Type 3 are isomorphic as graded rings if and only if $(A, \mathbf{b})=\left(A^{\prime}, \mathbf{b}^{\prime}\right)$. In other words, when $n \geq 3$, two toric manifolds of Type 3 are isomorphic as varieties if and only if their cohomology rings are isomorphic as graded rings.

Proof. The matrix $A$ of Type 3 is determined by $a$, the $(1, n)$-entry, in Proposition 3.2. Let $A$ and $A^{\prime}$ be the matrices of Type 3 determined by $a$ and $a^{\prime}$, respectively.

Suppose that $a \neq a^{\prime}$ and there is a graded ring isomorphism $\varphi: H^{*}(X(A, \mathbf{b})) \rightarrow$ $H^{*}\left(X\left(A^{\prime}, \mathbf{b}^{\prime}\right)\right)$. We shall deduce a contradiction. Since $\varphi$ is an isomorphism, it follows from Corollary 4.2 that

$$
|\operatorname{det} A|=|1+a|=\left|1+a^{\prime}\right|=\left|\operatorname{det} A^{\prime}\right| .
$$

Hence, we have $a+a^{\prime}=-2$. We express $\varphi\left(x_{1}\right)$ and $\varphi\left(x_{n}\right)$ as

$$
\varphi\left(x_{1}\right) \equiv \sum_{i=1}^{n} r_{i} x_{i} \quad(\bmod x) \quad \text { and } \quad \varphi\left(x_{n}\right) \equiv \sum_{i=1}^{n} q_{i} x_{i} \quad(\bmod x)
$$

where $q_{i}$ and $r_{i}$ are integers satisfying

$$
\operatorname{gcd}\left(q_{1}, \ldots, q_{n}\right)=\operatorname{gcd}\left(r_{1}, \ldots, r_{n}\right)=1 .
$$

Since $\varphi(x)= \pm x$ by Lemma 4.3, we have

$$
0=\varphi\left(x_{1}\left(x_{1}+a x_{n}+x\right)\right) \equiv\left(\sum_{i=1}^{n} r_{i} x_{i}\right)\left(\sum_{j=1}^{n}\left(a q_{j}+r_{j}\right) x_{j}\right) \quad(\bmod x) .
$$


Here

$$
x_{1}^{2} \equiv-a^{\prime} x_{n} x_{1} \quad(\bmod x), \quad x_{i}^{2} \equiv x_{i} x_{i-1} \quad(\bmod x) \quad(2 \leq i \leq n)
$$

in $H^{*}\left(X\left(A^{\prime}, \mathbf{b}^{\prime}\right)\right)$. Plugging these into (5.5) and looking at the coefficients of $x_{i} x_{j}$ $(n \geq i>j \geq 1)$, we see that

$$
\begin{array}{lll}
r_{n}\left(a q_{1}+r_{1}\right)+r_{1}\left(a q_{n}+r_{n}\right)-r_{1}\left(a q_{1}+r_{1}\right) a^{\prime}=0, & \\
r_{i}\left(a q_{i-1}+r_{i-1}\right)+r_{i-1}\left(a q_{i}+r_{i}\right)+r_{i}\left(a q_{i}+r_{i}\right)=0 & (2 \leq i \leq n), \\
r_{i}\left(a q_{j}+r_{j}\right)+r_{j}\left(a q_{i}+r_{i}\right)=0 & ((i, j) \neq(n, 1), i-j \geq 2) .
\end{array}
$$

Let $p$ be a divisor of the integer $a$. Since $a+a^{\prime}=-2$, the equations above reduce to

$$
\begin{array}{rll}
2 r_{1}\left(r_{n}+r_{1}\right) \equiv 0 & (\bmod p), & \\
r_{i}\left(2 r_{i-1}+r_{i}\right) \equiv 0 & (\bmod p) & (2 \leq i \leq n), \\
2 r_{i} r_{j} \equiv 0 & (\bmod p) & ((i, j) \neq(n, 1), i-j \geq 2) .
\end{array}
$$

Suppose that $a$ is even. Then $r_{i}$ is even for $2 \leq i \leq n$ by (5.7) and hence $r_{1}$ is odd by (5.4). Therefore $2 r_{1}\left(r_{n}+r_{1}\right) \not \equiv 0(\bmod 4)$. This together with (5.6) shows that $a$ is not divisible by four and hence $a \equiv 2(\bmod 4)$. Since $a+a^{\prime}=-2, a^{\prime}$ is also even and the same argument as for $a$ shows that $a^{\prime} \equiv 2(\bmod 4)$. However, these contradict the assumption $a+a^{\prime}=-2$.

Therefore, both $a$ and $a^{\prime}$ must be odd. Suppose $|a| \geq 3$ and let $p$ be an odd prime integer which divides $a$. Then there exists $r_{i}$ such that $r_{i} \not \equiv 0(\bmod p)$ from (5.4) and then $r_{i-1} \not \equiv 0(\bmod p)$ from (5.6) and (5.7), where $r_{0}=r_{n}$. Therefore $r_{i} \not \equiv 0$ $(\bmod p)$ for any $i$. However, this contradicts (5.8) when $n \geq 4$. When $n=3$, (5.6) and (5.7) have a nontrivial common solution only when $p=5$. Since $a$ is odd, it follows that $a= \pm 5^{u}$ for some $u \geq 1$. Therefore $\left|a^{\prime}\right| \geq 3$ since $a+a^{\prime}=-2$. Then the same argument as for $a$ shows that $a^{\prime}= \pm 5^{v}$ for some $v \geq 1$. However, these contradict the assumption $a+a^{\prime}=-2$. Thus, $|a|=\left|a^{\prime}\right|=1$. However, this again contradicts $a+a^{\prime}=-2$ because $a \neq a^{\prime}$.

This completes the proof of the proposition.

5.5. Isomorphism classes and determinant. We have studied isomorphism classes of cohomology rings in each type and end up with the following proposition.

Proposition 5.6. If $H^{*}(X(A, \mathbf{b}))$ and $H^{*}\left(X\left(A^{\prime}, \mathbf{b}^{\prime}\right)\right)$ are isomorphic as graded rings, then $\operatorname{det} A=\operatorname{det} A^{\prime}$, and the converse holds unless the value of the determinant is one.

Proof. We note that the proof of Proposition 5.5 holds for all values of $a$, i.e. even for $a=0, \pm 1$, and the matrix $A$ in Type 3 is of Type 0,1 and 2 when $a=-1,0$ and 1 . We also know that there is only one isomorphism class in the cohomology rings in Type 0 and 2 by Lemmas 5.1 and 5.4 and if $H^{*}(X(A, \mathbf{b}))$ and $H^{*}\left(X\left(A^{\prime}, \mathbf{b}^{\prime}\right)\right)$ are isomorphic as graded rings, then $|\operatorname{det} A|=\left|\operatorname{det} A^{\prime}\right|$ by Corollary 4.2. Therefore, it suffices to prove that $H^{*}(X(A, \mathbf{b}))$ for $A$ with $a=-2$ in Type 3 is not isomorphic to any cohomology ring in Type 1.

If $H^{*}(X(A, \mathbf{b}))$ above is isomorphic to a cohomology ring $H^{*}\left(X\left(A^{\prime}, \mathbf{b}^{\prime}\right)\right)$ in Type 1 , then the quotient ring $H^{*}(X(A, \mathbf{b})) /(x)$ is isomorphic to $H^{*}\left(X\left(A^{\prime}, \mathbf{b}^{\prime}\right)\right) /(x)$ because any isomorphism sends $x$ to $x$ up to sign by Lemma 4.3. The element $x_{1}$ in $H^{*}\left(X\left(A^{\prime}, \mathbf{b}^{\prime}\right)\right) /(x)$ is nonzero but its square vanishes. Therefore, it suffices to show 
that if $\alpha$ is a nonzero degree two element in $H^{*}(X(A, \mathbf{b})) /(x)$, then $\alpha^{2}$ does not vanish in $H^{*}(X(A, \mathbf{b})) /(x)$. Write $\alpha=\sum_{i=1}^{n} c_{i} x_{i}$ with integers $c_{i}$. Since $x_{1}^{2}=2 x_{n} x_{1}$ and $x_{i}^{2}=x_{i} x_{i-1}$ for $2 \leq i \leq n$ in $H^{*}(X(A, \mathbf{b})) /(x)$, we have

$$
\begin{aligned}
\left(\sum_{i=1}^{n} c_{i} x_{i}\right)^{2} & =\sum_{i=1}^{n} c_{i}^{2} x_{i}^{2}+2 \sum_{1 \leq j<i \leq n} c_{i} c_{j} x_{i} x_{j} \\
& =2\left(c_{1}^{2}+c_{n} c_{1}\right) x_{n} x_{1}+\sum_{i=2}^{n}\left(c_{i}^{2}+2 c_{i} c_{i-1}\right) x_{i} x_{i-1}+2 \sum_{\substack{i, j) \neq(n, 1) \\
i-j \geq 2}} c_{i} c_{j} x_{i} x_{j}
\end{aligned}
$$

Therefore, if $\left(\sum_{i=1}^{n} c_{i} x_{i}\right)^{2}=0$ in $H^{*}(X(A, \mathbf{b})) /(x)$, then we have

$c_{1}\left(c_{1}+c_{n}\right)=0, \quad c_{i}\left(c_{i}+2 c_{i-1}\right)=0(2 \leq i \leq n), \quad c_{i} c_{j}=0((i, j) \neq(n, 1), i-j \geq 2)$

since $x_{i} x_{j}(1 \leq j<i \leq n)$ are an additive basis of $H^{*}(X(A, \mathbf{b})) /(x)$. An elementary check shows that the equations above have only the trivial solution, proving the proposition.

\section{Smooth classification in Type 0}

We have seen that the cohomology rings of the toric manifolds of Type 0 are isomorphic to each other (Lemma 5.1). The purpose of this section is to prove the following proposition using the quotient construction of toric manifolds.

Proposition 6.1. All the toric manifolds of Type 0 are diffeomorphic to each other.

Remember that the matrix $[A \mid \mathbf{b}]$ in Type 0 is of the form

$$
\left[\begin{array}{cccccc}
1 & 0 & \ldots & 0 & -1 & b_{1} \\
-1 & 1 & \ldots & 0 & 0 & b_{2} \\
\vdots & \ddots & \ddots & \vdots & \vdots & \vdots \\
0 & 0 & \ldots & 1 & 0 & b_{n-1} \\
0 & 0 & \ldots & -1 & 1 & b_{n}
\end{array}\right] \text { with } \sum_{i=1}^{n} b_{i}=1
$$

We add the first row to the second row in $\left[-E_{n}|A| \mathbf{b}\right]$, and then add the second row to the third row in the new matrix. We repeat the process and end up with the matrix

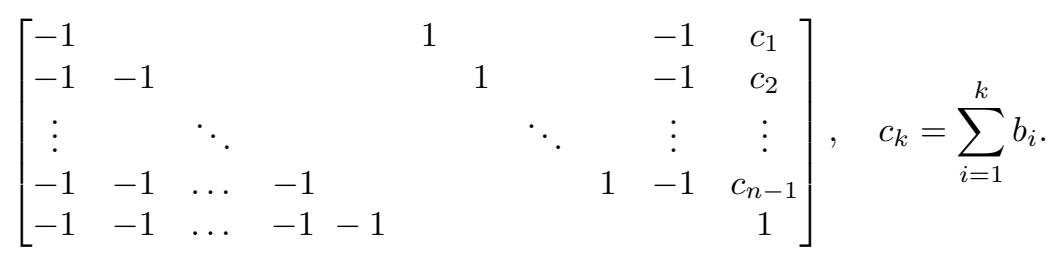

Since the fan of Type 0 is isomorphic to the fan determined by the matrix in (6.1), we henceforth consider the toric manifold associated with the matrix in (6.1), denoted by $X_{\mathbf{c}}$, where $\mathbf{c}=\left[c_{1}, \ldots, c_{n-1}\right]^{T}$. Note that

$$
\sum_{k=1}^{n-1} c_{k}=\sum_{i=1}^{n-1}(n-i) b_{i} .
$$


On the other hand, for a cyclic permutation $\sigma(i)=i+\ell$ on $[n]$, we have

$$
\begin{aligned}
& \sum_{i=1}^{n-1}(n-i) b_{\sigma(i)}-\sum_{i=1}^{n-1}(n-i) b_{i} \equiv-\sum_{i=1}^{n} i b_{\sigma(i)}-\left(-\sum_{i=1}^{n} i b_{i}\right) \\
\equiv & -\sum_{i=1}^{n} i b_{i+\ell}+\sum_{i=1}^{n} i b_{i} \equiv-\sum_{j=1}^{n}(j-\ell) b_{j}+\sum_{i=1}^{n} i b_{i} \\
\equiv & \ell \sum_{j=1}^{n} b_{j} \equiv \ell \quad(\bmod n) .
\end{aligned}
$$

Hence, we may restrict our attention to the toric manifolds determined by a vector $\mathbf{c}$ satisfying $\sum_{k=1}^{n-1} c_{k} \equiv 0(\bmod n)$.

Let us construct the toric manifold $X_{\mathbf{c}}$ associated with $\mathbf{c}$ by using the quotient construction explained in Section 2. Remember that $\mathcal{C}_{\mathrm{n}}$ is the simplicial complex whose minimal non-faces are $\{i, n+i\},\{i, 2 n+1\}$ for $1 \leq i \leq n$ and $\{n+1, \ldots, 2 n\}$. Letting $\left(z_{1}, \ldots, z_{n}, w_{1}, \ldots, w_{n}, w\right)$ be the coordinates of $\mathbb{C}^{2 n+1}$, we define

$$
Z=\bigcup_{i=1}^{n}\left\{z_{i}=w_{i}=0\right\} \cup\left\{w_{1}=\cdots=w_{n}=0\right\} \cup \bigcup_{i=1}^{n}\left\{z_{i}=w=0\right\} .
$$

Let $\lambda_{\mathbf{c}}:\left(\mathbb{C}^{*}\right)^{2 n+1} \rightarrow\left(\mathbb{C}^{*}\right)^{n}$ be the homomorphism determined by 6.1 , that is,

$$
\begin{aligned}
& \lambda_{\mathbf{c}}\left(g_{1}, \ldots, g_{n}, h_{1}, \ldots, h_{n}, h\right) \\
= & \left(g_{1}^{-1} h_{1} h_{n}^{-1} h^{c_{1}},\left(g_{1} g_{2}\right)^{-1} h_{2} h_{n}^{-1} h^{c_{2}}, \ldots,\left(g_{1} \cdots g_{n-1}\right)^{-1} h_{n-1} h_{n}^{-1} h^{c_{n-1}},\left(g_{1} \ldots g_{n}\right)^{-1} h\right) .
\end{aligned}
$$

Then the kernel of $\lambda_{\mathbf{c}}$ is given by

$\left\{\left(g_{1}, \ldots, g_{n}, g_{1} h_{n} h^{-c_{1}}, g_{1} g_{2} h_{n} h^{-c_{2}}, \ldots,\left(g_{1} \cdots g_{n-1}\right) h_{n} h^{-c_{n-1}}, h_{n}, h\right) \mid h=g_{1} \cdots g_{n}\right\}$.

Hence, we get

$$
X_{\mathbf{c}}=\left(\mathbb{C}^{2 n+1} \backslash Z\right) / \operatorname{ker} \lambda_{\mathbf{c}}
$$

We set

$$
X_{\mathbf{c}}^{-}=X_{\mathbf{c}} \cap\{w \neq 0\}, \quad X_{\mathbf{c}}^{+}=X_{\mathbf{c}} \cap \bigcap_{i=1}^{n}\left\{z_{i} \neq 0\right\} .
$$

We have $X_{\mathbf{c}}=X_{\mathbf{c}}^{-} \cup X_{\mathbf{c}}^{+}$.

Proof of Proposition 6.1. It is sufficient to prove that

$$
X_{\mathbf{c}} \text { is diffeomorphic to } X_{\mathbf{0}} \text {, where } \mathbf{0}:=[0, \ldots, 0]^{T} \in \mathbb{Z}^{n-1},
$$

since it means that $X(A, \mathbf{b})$ is diffeomorphic to $X\left(A, \mathbf{b}^{\prime}\right)$, where $\mathbf{b}=\left[b_{1}, \ldots, b_{n}\right]^{T}$ with $\sum_{i=1}^{n} b_{i}=1$ and $\mathbf{b}^{\prime}=[0, \ldots, 0,1]^{T}$.

We consider the diffeomorphism $\varphi_{\mathbf{c}}: X_{\mathbf{c}}^{-} \rightarrow X_{\mathbf{0}}^{-}$given by

$$
\varphi_{\mathbf{c}}\left(\left[z_{1}, \ldots, z_{n}, w_{1}, \ldots, w_{n}, w\right]_{\mathbf{c}}\right)=\left[z_{1}, \ldots, z_{n}, w^{c_{1}} w_{1}, \ldots, w^{c_{n-1}} w_{n-1}, w_{n}, w\right]_{\mathbf{0}}
$$

where [ ] $\mathbf{c}_{\mathbf{c}}$ and [ $]_{\mathbf{0}}$ are points in $X_{\mathbf{c}}$ and $X_{\mathbf{0}}$, respectively, and try to extend it to a diffeomorphism from $X_{\mathbf{c}}$ to $X_{\mathbf{0}}$. We note that $X_{\mathbf{c}}^{+}$is diffeomorphic to $\mathbb{P}^{n-1} \times \mathbb{C}$ via the map

$$
\begin{aligned}
& \psi_{\mathbf{c}}\left(\left[z_{1}, \ldots, z_{n}, w_{1}, \ldots, w_{n}, w\right]_{\mathbf{c}}\right) \\
& =\left(\left[z_{1}^{-1}\left(z_{1} \cdots z_{n}\right)^{c_{1}} w_{1}, \ldots,\left(z_{1} \cdots z_{n-1}\right)^{-1}\left(z_{1} \cdots z_{n}\right)^{c_{n-1}} w_{n-1}, w_{n}\right],\left(z_{1} \cdots z_{n}\right)^{-1} w\right) .
\end{aligned}
$$


Similarly, $X_{0}^{+}$is diffeomorphic to $\mathbb{P}^{n-1} \times \mathbb{C}$ via the map

$$
\begin{aligned}
& \psi_{\mathbf{0}}\left(\left[z_{1}, \ldots, z_{n}, w_{1}, \ldots, w_{n}, w\right]_{\mathbf{0}}\right) \\
& \quad=\left(\left[z_{1}^{-1} w_{1}, \ldots,\left(z_{1} \cdots z_{n-1}\right)^{-1} w_{n-1}, w_{n}\right],\left(z_{1} \cdots z_{n}\right)^{-1} w\right) .
\end{aligned}
$$

Therefore, $\psi_{\mathbf{0}} \circ \varphi_{\mathbf{c}} \circ \psi_{\mathbf{c}}^{-1}$ is a self-diffeomorphism of $\mathbb{P}^{n-1} \times \mathbb{C}^{*}$ given by

$$
\begin{aligned}
& \psi_{\mathbf{0}} \circ \varphi_{\mathbf{c}} \circ \psi_{\mathbf{c}}^{-1}\left(\left[w_{1}, \ldots, w_{n}\right], w\right) \\
= & \psi_{\mathbf{0}} \circ \varphi_{\mathbf{c}}\left(\left[1, \ldots, 1, w_{1}, \ldots, w_{n}, w\right]_{\mathbf{c}}\right) \\
= & \psi_{\mathbf{0}}\left(\left[1, \ldots, 1, w^{c_{1}} w_{1}, \ldots, w^{c_{n-1}} w_{n-1}, w_{n}, w\right]_{\mathbf{0}}\right) \\
= & \left(\left[w^{c_{1}} w_{1}, \ldots, w^{c_{n-1}} w_{n-1}, w_{n}\right], w\right),
\end{aligned}
$$

that is,

$$
\left(\left[w_{1}, \ldots, w_{n}\right], w\right) \longmapsto\left(\left[w^{c_{1}} w_{1}, \ldots, w^{c_{n-1}} w_{n-1}, w_{n}\right], w\right) .
$$

If this self-diffeomorphism of $\mathbb{P}^{n-1} \times \mathbb{C}^{*}$ extends to a self-diffeomorphism of $\mathbb{P}^{n-1} \times \mathbb{C}$, then we are done. But this is impossible. To avoid this difficulty, we restrict the map (6.2) to $\mathbb{P}^{n-1} \times\left(\mathbb{C} \backslash \operatorname{Int} D^{2}\right)$, where $D^{2}$ is the unit disk of $\mathbb{C}$, and find its extension to a self-diffeomorphism of $\mathbb{P}^{n-1} \times \mathbb{C}$.

We regard the map (6.2) as the homomorphism

$$
\rho: \mathbb{C}^{*} \rightarrow \operatorname{PU}(n), \quad w \mapsto \operatorname{diag}\left(w^{c_{1}}, \ldots, w^{c_{n-1}}, 1\right)
$$

where $\mathrm{PU}(n)$ denotes the quotient of the unitary group $\mathrm{U}(n)$ by its center and $\operatorname{diag}\left(\right.$ ) means a diagonal matrix. It suffices to show that $\rho$ restricted to $\left(\mathbb{C} \backslash \operatorname{Int} D^{2}\right)$, denoted by $\bar{\rho}$, extends to a continuous map from $\mathbb{C}$ to $\mathrm{PU}(n){ }^{3}$ For every integer $m$,

$$
\operatorname{diag}\left(w^{c_{1}}, \ldots, w^{c_{n-1}}, 1\right)=\operatorname{diag}\left(w^{c_{1}+m}, \ldots, w^{c_{n-1}+m}, w^{m}\right) \quad \text { in } \mathrm{PU}(n) .
$$

Since $\sum_{k=1}^{n-1} c_{k} \equiv 0(\bmod n)$, this implies that the homomorphism $\rho$ above factors through the special unitary group $\mathrm{SU}(n)$. Since $\mathrm{SU}(n)$ is simply connected, the restriction of $\bar{\rho}$ to the boundary $S^{1}$ of $D^{2}$ is null homotopic. Therefore, $\bar{\rho}$ extends to a map $\mathbb{C} \rightarrow \mathrm{PU}(n)$ continuously.

\section{Smooth Classification in Type 2}

We have seen that the cohomology rings of the toric manifolds of Type 2 are isomorphic to each other (Lemma 5.4). The purpose of this section is to prove the following proposition using moment-angle manifolds associated with an $n$-cube with one vertex cut, which is a deformation retract of $\mathbb{C}^{2 n+1} \backslash Z$ used in the previous section.

Proposition 7.1. All the toric manifolds of Type 2 are diffeomorphic to each other.

First, let us review the definition of the moment-angle manifold $\mathcal{Z}_{P}$ obtained from a simple polytope $P$. Consider an $n$-dimensional simple polytope

$$
P=\left\{\mathbf{x}=\left(x_{1}, \ldots, x_{n}\right) \in \mathbb{R}^{n} \mid\left\langle\mathbf{n}_{i}, \mathbf{x}\right\rangle+\gamma_{i} \geq 0 \text { for } i=1, \ldots, m\right\}
$$

\footnotetext{
${ }^{3}$ Let $M$ and $N$ be smooth manifolds and $f: M \rightarrow N$ be a continuous map. If $f$ is smooth on a closed subset $A$ in $M$, then the map $f$ restricted to $A$ extends to a smooth map. We refer the reader to [14 Theorem 2.23] for the proof. We apply this theorem for $M=\mathbb{C}, N=\mathrm{PU}(n)$ and $A=\mathbb{C} \backslash \operatorname{Int} D^{2}$.
} 
where $\mathbf{n}_{i} \in \mathbb{R}^{n}, \gamma_{i} \in \mathbb{R}$ and $\langle$,$\rangle denotes the standard scalar product on \mathbb{R}^{n}$. We assume that $m$ agrees with the number of facets of $P$. Define the map

$$
\iota_{P}: \mathbb{R}^{n} \rightarrow \mathbb{R}^{m}, \quad \iota_{P}(\mathbf{x})=\left(\left\langle\mathbf{n}_{1}, \mathbf{x}\right\rangle+\gamma_{1}, \ldots,\left\langle\mathbf{n}_{m}, \mathbf{x}\right\rangle+\gamma_{m}\right) .
$$

It embeds $P$ into $\mathbb{R}_{\geq 0}^{m}$. Then the moment-angle manifold $\mathcal{Z}_{P}$ associated with $P$ is defined to be the fiber product of the commutative diagram

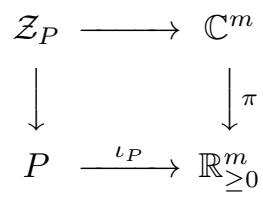

where $\pi\left(z_{1}, \ldots, z_{m}\right)=\left(\left|z_{1}\right|^{2}, \ldots,\left|z_{m}\right|^{2}\right)$. We note that $\mathcal{Z}_{P}$ is invariant under the standard action of $\left(S^{1}\right)^{m}$ on $\mathbb{C}^{m}$. The moment-angle manifold associated with an $n$-cube is the product of $n$ copies of 3 -spheres.

Let us consider the polytope $P$ presented as follows:

$$
P=\left\{\left(x_{1}, \ldots, x_{n}\right) \in \mathbb{R}^{n} \mid 0 \leq x_{i} \leq 1, \sum_{i=1}^{n} x_{i} \leq n-\frac{1}{2}\right\} .
$$

Then $P$ is an $n$-cube with one vertex cut $\mathrm{vc}\left(I^{n}\right)$ as a manifold with corners, so the boundary complex of the simplicial polytope dual to $P$ is isomorphic to our simplicial complex $\mathcal{C}_{\mathrm{n}}$. One can see that the moment-angle manifold $\mathcal{Z}_{P}$ associated with $P$ can be described as

$$
\left\{\left.\left(z_{1}, \ldots, z_{n}, w_{1}, \ldots, w_{n}, w\right) \in \mathbb{C}^{2 n+1}|| z_{i}\right|^{2}+\left|w_{i}\right|^{2}=1, \sum_{i=1}^{n}\left|w_{i}\right|^{2}=|w|^{2}+\frac{1}{2}\right\} .
$$

As is well-known, $\mathcal{Z}_{P}$ is a deformation retract of $\mathbb{C}^{2 n+1} \backslash Z$, where

$$
Z=\bigcup_{i=1}^{n}\left\{z_{i}=w_{i}=0\right\} \cup\left\{w_{1}=\cdots=w_{n}=0\right\} \cup \bigcup_{i=1}^{n}\left\{z_{i}=w=0\right\}
$$

as before.

Suppose that $(A, \mathbf{b})$ is of Type 2 . We define a homomorphism $\lambda_{A}:\left(\mathbb{C}^{*}\right)^{2 n+1} \rightarrow$ $\left(\mathbb{C}^{*}\right)^{n}$ by

$$
\lambda_{A}\left(g_{1}, \ldots, g_{n}, h_{1}, \ldots, h_{n}, h\right)=\left(g_{1}^{-1} h_{1} h_{n}^{a_{1}} h^{b_{1}}, g_{2}^{-1} h_{1}^{a_{2}} h_{2} g^{b_{2}}, \ldots, g_{n}^{-1} h_{n-1}^{a_{n}} h_{n} h^{b_{n}}\right) .
$$

Then

$$
\left.\operatorname{ker} \lambda_{A}=\left\{h_{1} h_{n}^{a_{1}} h^{b_{1}}, h_{1}^{a_{2}} h_{2} h^{b_{2}}, \ldots, h_{n-1}^{a_{n}} h_{n} h^{b_{n}}, h_{1}, \ldots, h_{n}, h\right)\right\}
$$

and the quotient construction of toric manifolds tells us that

$$
X(A, \mathbf{b})=\left(\mathbb{C}^{2 n+1} \backslash Z\right) / \operatorname{ker} \lambda_{A} .
$$

The following proposition is a real analog of this quotient construction. It is wellknown for experts, but we shall give a proof in Appendix for the convenience of the reader.

Proposition 7.2. The toric manifold $X(A, \mathbf{b})$ of Type 2 is $\left(S^{1}\right)^{n}$-equivariantly diffeomorphic to $\mathcal{Z}_{P} / \operatorname{ker} \lambda_{A}^{S}$, where $\lambda_{A}^{S}$ is the restriction of $\lambda_{A}$ to $\left(S^{1}\right)^{2 n+1}$. 
We prepare some notations and a lemma we need later. Define the matrix $R$ by

$$
R=A^{-1}+\frac{1}{2} J
$$

where $J$ is the $n \times n$ matrix all of whose entries are 1 . One can easily check that every component of $A^{-1}$ is either $1 / 2$ or $-1 / 2$, so the matrix $R$ is an integer matrix. Let $\mathbf{r}_{i}$ be the $i$ th row of $R$ and $\mathbf{u}_{i}$ be the $i$ th row of $A$. We write $\mathbf{r}_{i}=\left[r_{i 1}, r_{i 2}, \ldots, r_{i n}\right]$ and $\mathbf{u}_{i}=\left[u_{i 1}, \ldots, u_{i n}\right]$. Set $\mathbf{e}_{i}^{\prime}=[0, \ldots, 0,1,0, \ldots, 0]$, the vector with a 1 in the $i$ th coordinate and 0 's elsewhere, and $\mathbf{1}:=[1, \ldots, 1]$.

Lemma 7.3. Under the notations above, we obtain the following:

(1) $\sum_{j=1}^{n} r_{i j} \mathbf{u}_{j}=\frac{1}{2} \sum_{j=1}^{n} \mathbf{u}_{j}+\mathbf{e}_{i}^{\prime}$;

(2) $\sum_{j=1}^{n} r_{i j} b_{j}=\frac{1}{2}\left(1+\sum_{j=1}^{n} b_{j}\right)$; and

(3) $\sum_{j=1}^{n} u_{i j} \mathbf{r}_{j}-b_{i} \mathbf{1}=\mathbf{e}_{i}^{\prime}$,

where $b_{i}$ is the ith entry of $\mathbf{b}$, that is, $b_{i}=\frac{1}{2}\left(1+a_{i}\right)$ for $1 \leq i \leq n$.

Proof. From (7.4), we have

$$
R A=\frac{1}{2} J A+E_{n}
$$

Then we obtain (1) by comparing the rows on both sides of (7.5).

Multiplying both sides of (7.4) by $\mathbf{b}$ from the right, we have

$$
R\left[\begin{array}{c}
b_{1} \\
\vdots \\
b_{n}
\end{array}\right]=A^{-1}\left[\begin{array}{c}
b_{1} \\
\vdots \\
b_{n}
\end{array}\right]+\frac{1}{2} \sum_{j=1}^{n} b_{j}\left[\begin{array}{c}
1 \\
\vdots \\
1
\end{array}\right]
$$

Then, noting $A \mathbf{1}^{T}=2 \mathbf{b}$, we obtain (2) by comparing the rows on both sides of (7.6).

From (7.4), we have

$$
A R-\frac{1}{2} A J=E_{n}
$$

Since $\sum_{j=1}^{n} u_{i j}=1+a_{i}=2 b_{i}$, we obtain (3) by comparing the rows on both sides of (7.7).

We look at two parts of $\mathcal{Z}_{P} / \operatorname{ker} \lambda_{A}^{S}$ :

$$
\left(\mathcal{Z}_{P} / \operatorname{ker} \lambda_{A}^{S}\right) \cap\{|w|<1 / \sqrt{2}\} \quad \text { and } \quad\left(\mathcal{Z}_{P} / \operatorname{ker} \lambda_{A}^{S}\right) \cap\{w \neq 0\} .
$$

Note that if $|w|<1 / \sqrt{2}$, then $z_{i} \neq 0$ for every $i=1, \ldots, n$ by (7.2). Write

$$
\boldsymbol{z}^{\mathbf{c}}=\prod_{j=1}^{n} z_{j}^{c_{j}} \text { and } \boldsymbol{h}^{c}=\prod_{j=1}^{n} h_{j}^{c_{j}}
$$

for $\mathbf{c}=\left[c_{1}, \ldots, c_{n}\right]$. It follows from (7.3) that

$$
\operatorname{ker} \lambda_{A}^{S}=\left\{\left(\boldsymbol{h}^{\mathbf{u}_{1}} h^{b_{1}}, \ldots, \boldsymbol{h}^{\mathbf{u}_{n}} h^{b_{n}}, h_{1}, \ldots, h_{n}, h\right) \in\left(S^{1}\right)^{2 n+1} \mid h_{i}, h \in S^{1}\right\} .
$$

We define

$$
\tilde{L}=\left\{\left.\left(v_{1}, \ldots, v_{n}, u\right) \in \mathbb{C}^{n+1}\left|\sum_{i=1}^{n}\right| v_{i}|=| u\right|^{2}+\frac{1}{2},|u|<\frac{1}{\sqrt{2}}\right\} \text { and } L=\tilde{L} / S^{1},
$$


where the action of $S^{1}$ on $\tilde{L}$ is given by

$$
g \cdot\left(v_{1}, \ldots, v_{n}, u\right)=\left(g v_{1}, \ldots, g v_{n}, g^{-2} u\right) \text { for } g \in S^{1} .
$$

Lemma 7.4. The map $\varphi_{A}:\left(\mathbb{C}^{*}\right)^{n} \times \mathbb{C}^{n+1} \rightarrow \tilde{L}$ defined by

$$
\varphi_{A}\left(z_{1}, \ldots, z_{n}, w_{1}, \ldots, w_{n}, w\right)=\left(\left(\frac{\boldsymbol{z}^{\mathbf{r}_{1}}}{\left|\boldsymbol{z}^{\mathbf{r}_{1}}\right|}\right)^{-1} w_{1}, \ldots,\left(\frac{\boldsymbol{z}^{\mathbf{r}_{n}}}{\left|\boldsymbol{z}^{\mathbf{r}_{n}}\right|}\right)^{-1} w_{n}, \frac{\boldsymbol{z}^{\mathbf{1}}}{\left|\boldsymbol{z}^{\mathbf{1}}\right|} w\right)
$$

induces a diffeomorphism $\hat{\varphi}_{A}:\left(\mathcal{Z}_{P} / \operatorname{ker} \lambda_{A}^{S}\right) \cap\{|w|<1 / \sqrt{2}\} \rightarrow L$.

Proof. We first show that $\varphi_{A}$ induces a smooth map

$$
\hat{\varphi}_{A}:\left(\mathcal{Z}_{P} / \operatorname{ker} \lambda_{A}^{S}\right) \cap\{|w|<1 / \sqrt{2}\} \rightarrow L .
$$

By (7.8) it is sufficient to show that for each $\left(h_{1}, \ldots, h_{n}, h\right) \in\left(S^{1}\right)^{n+1}$, there exists $t \in S^{1}$ such that

$$
\begin{aligned}
& \varphi_{A}\left(\boldsymbol{h}^{\mathbf{u}_{1}} h^{b_{1}} z_{1}, \ldots, \boldsymbol{h}^{\mathbf{u}_{n}} h^{b_{n}} z_{n}, h_{1} w_{1}, \ldots, h_{n} w_{n}, h w\right) \\
& =\left(t\left(\frac{\boldsymbol{z}^{\mathbf{r}_{1}}}{\left|\boldsymbol{z}^{\mathbf{r}_{1}}\right|}\right)^{-1} w_{1}, \ldots, t\left(\frac{\boldsymbol{z}^{\mathbf{r}_{n}}}{\left|\boldsymbol{z}^{\mathbf{r}_{n}}\right|}\right)^{-1} w_{n}, t^{-2} \frac{\boldsymbol{z}^{\mathbf{1}}}{\left|\boldsymbol{z}^{\mathbf{1}}\right|} w\right) .
\end{aligned}
$$

Since we have $\left|h_{i}\right|=|h|=1$ for every $i=1, \ldots, n$, the $i$ th coordinate of the left hand side of (7.11) is equal to

$$
\begin{aligned}
\left(\prod_{j=1}^{n}\left(\boldsymbol{h}^{\mathbf{u}_{j}} h^{b_{j}} z_{j} /\left|z_{j}\right|\right)^{r_{i j}}\right)^{-1} h_{i} w_{i} & =\left(\boldsymbol{h}^{\sum_{j=1}^{n} r_{i j} \mathbf{u}_{j}} h^{\sum_{j=1}^{n} r_{i j} b_{j}} \frac{\boldsymbol{z}^{\mathbf{r}_{i}}}{\mid \boldsymbol{z}^{\mathbf{r}_{i} \mid}}\right)^{-1} h_{i} w_{i} \\
& =\left(\boldsymbol{h}^{\frac{1}{2} \sum_{j=1}^{n} \mathbf{u}_{j}} h_{i} h^{\frac{1}{2}\left(1+\sum_{j=1}^{n} b_{j}\right)} \frac{\boldsymbol{z}^{\mathbf{r}_{i}}}{\mid \boldsymbol{z}^{\mathbf{r}_{i} \mid}}\right)^{-1} h_{i} w_{i} \\
& =\left(\boldsymbol{h}^{\frac{1}{2} \sum_{j=1}^{n} \mathbf{u}_{j}} h^{\frac{1}{2}\left(1+\sum_{j=1}^{n} b_{j}\right)}\right)^{-1}\left(\frac{\boldsymbol{z}^{\mathbf{r}_{i}}}{\mid \boldsymbol{z}^{\mathbf{r}_{i} \mid}}\right)^{-1} w_{i},
\end{aligned}
$$

where the second equality follows from (1) and (2) in Lemma 7.3, The $(n+1)$ th coordinate of the left hand side of (7.11) is equal to

$$
\left(\prod_{j=1}^{n}\left(\boldsymbol{h}^{\mathbf{u}_{j}} h^{b_{j}} z_{j} /\left|z_{j}\right|\right)\right) h w=\left(\boldsymbol{h}^{\sum_{j=1}^{n} \mathbf{u}_{j}} h^{1+\sum_{j=1}^{n} b_{j}}\right) \frac{\boldsymbol{z}^{\mathbf{1}}}{\left|\boldsymbol{z}^{\mathbf{1}}\right|} w .
$$

Hence, (7.11) holds for $t=\left(\boldsymbol{h}^{\frac{1}{2} \sum_{j=1}^{n} \mathbf{u}_{j}} h^{\frac{1}{2}\left(1+\sum_{j=1}^{n} b_{j}\right)}\right)^{-1} \in S^{1}$.

Let us show the injectivity of $\hat{\varphi}_{A}$. Suppose that

$$
\varphi_{A}\left(z_{1}, \ldots, z_{n}, w_{1}, \ldots, w_{n}, w\right)=\varphi_{A}\left(z_{1}^{\prime}, \ldots, z_{n}^{\prime}, w_{1}^{\prime}, \ldots, w_{n}^{\prime}, w^{\prime}\right) \text { in } L,
$$

that is, there is an element $t \in S^{1}$ such that

$$
\begin{aligned}
& \left(\left(\frac{\boldsymbol{z}^{\mathbf{r}_{1}}}{\left|\boldsymbol{z}^{\mathbf{r}_{1}}\right|}\right) w_{1}^{\prime}, \ldots,\left(\frac{\boldsymbol{z}^{\prime \mathbf{r}_{n}}}{\left|\boldsymbol{z}^{\prime \mathbf{r}_{n}}\right|}\right) w_{n}^{\prime},\left(\frac{\boldsymbol{z}^{\prime \mathbf{1}}}{\left|\boldsymbol{z}^{\prime \mathbf{1}}\right|}\right) w^{\prime}\right) \\
= & \left(t\left(\frac{\boldsymbol{z}^{\mathbf{r}_{1}}}{\left|\boldsymbol{z}^{\mathbf{r}_{1}}\right|}\right) w_{1}, \ldots, t\left(\frac{\boldsymbol{z}^{\mathbf{r}_{n}}}{\left|\boldsymbol{z}^{\mathbf{r}_{n}}\right|}\right) w_{n}, t^{-2}\left(\frac{\boldsymbol{z}^{\mathbf{1}}}{\left|\boldsymbol{z}^{\mathbf{1}}\right|}\right) w\right) .
\end{aligned}
$$

We set $g_{i}=\left(\frac{z_{i}^{\prime}}{\left|z_{i}^{\prime}\right|}\right)^{-1}\left(\frac{z_{i}}{\left|z_{i}\right|}\right)$ and $\boldsymbol{g}^{\mathbf{c}}=\prod_{j=1}^{n} g_{j}^{c_{j}}$ for $\mathbf{c}=\left[c_{1}, \ldots, c_{n}\right]$. Then

$$
w_{i}^{\prime}=t \boldsymbol{g}^{\mathbf{r}_{i}} w_{i} \text { and } w^{\prime}=t^{-2} \boldsymbol{g}^{-\mathbf{1}} w
$$


from (7.12). Setting $h_{i}=t \boldsymbol{g}^{\mathbf{r}_{i}}$ and $h=t^{-2} \boldsymbol{g}^{-\mathbf{1}}$, we obtain

$$
\boldsymbol{h}^{\mathbf{u}_{i}} h^{b_{i}}=\left(\prod_{j=1}^{n} h_{j}^{u_{i j}}\right) h^{b_{i}}=\left(t^{\sum_{j=1}^{n} u_{i j}} \boldsymbol{g}^{\sum_{j=1}^{n} u_{i j} \mathbf{r}_{j}}\right)\left(t^{-2 b_{i}} \boldsymbol{g}^{-b_{i} \mathbf{1}}\right)=g_{i},
$$

where the last equality above follows from $\sum_{j=1}^{n} u_{i j}=1+a_{i}=2 b_{i}$ and (3) in Lemma 7.3. Therefore, $\left(g_{1}, \ldots, g_{n}, h_{1}, \ldots, h_{n}, h\right) \in \operatorname{ker} \lambda_{A}^{S}$, which implies that $\hat{\varphi}_{A}$ is injective.

For $\left(v_{1}, \ldots, v_{n}, u\right) \in \tilde{L},\left(z_{1}, \ldots, z_{n}, v_{1}, \ldots, v_{n}, u\right)$ with $z_{i}=\sqrt{1-\left|v_{i}\right|^{2}}$ is an element of $\mathcal{Z}_{P}$ and $\varphi_{A}\left(z_{1}, \ldots, z_{n}, v_{1}, \ldots, v_{n}, u\right)=\left(v_{1}, \ldots, v_{n}, u\right)$, which proves that $\hat{\varphi}_{A}$ is surjective.

Thus, $\hat{\varphi}$ is smooth and bijective. The inverse of $\hat{\varphi}$ is induced from the map sending $\left(v_{1}, \ldots, v_{n}, u\right) \in \tilde{L}$ to $\left(z_{1}, \ldots, z_{n}, v_{1}, \ldots, v_{n}, u\right) \in \mathcal{Z}_{P}$ with $z_{i}=\sqrt{1-\left|v_{i}\right|^{2}}$, so it is also smooth. Therefore, $\hat{\varphi}$ is a diffeomorphism.

Suppose that the matrix $A$ has at least two $(-1)$ 's in $\left\{a_{1}, \ldots, a_{n}\right\}$. We consider the indices of $a_{i}$ 's modulo $n$ as usual. Since the number of 1 's in $\left\{a_{1}, \ldots, a_{n}\right\}$ is odd, there exist indices $i<j$ such that

$$
a_{i}=-1, a_{i+1}=\cdots=a_{j-1}=1, a_{j}=-1, \text { and } j-i \text { is even. }
$$

Set

$$
a_{k}^{\prime}= \begin{cases}1 & \text { for } i \leq k \leq j \\ a_{k} & \text { otherwise }\end{cases}
$$

and denote by $A^{\prime}$ the matrix of Type 2 associated with $\left\{a_{1}^{\prime}, \ldots, a_{n}^{\prime}\right\}$. We will use the prime symbol to represent notations corresponding to $A^{\prime}$.

Lemma 7.5. The map $f: \mathbb{C}^{2 n} \times \mathbb{C}^{*} \rightarrow \mathbb{C}^{2 n} \times \mathbb{C}^{*}$ defined by

$$
\begin{aligned}
& f\left(z_{1}, \ldots, z_{n}, w_{1}, \ldots, w_{n}, w\right) \\
& =\left(z_{1}, \ldots, z_{i-1}, \frac{w}{|w|} \bar{z}_{i},\left(\frac{w}{|w|}\right)^{2} \bar{z}_{i+1}, \ldots,\left(\frac{w}{|w|}\right)^{2} \bar{z}_{j-1}, \frac{w}{|w|} z_{j},\right. \\
& \left.\quad z_{j+1}, \ldots, z_{n}, w_{1}, \ldots, w_{i-1}, \bar{w}_{i}, \ldots, \bar{w}_{j-1}, w_{j}, \ldots, w_{n}, w\right)
\end{aligned}
$$

induces a diffeomorphism $\hat{f}:\left(\mathcal{Z}_{P} / \operatorname{ker} \lambda_{A}^{S}\right) \cap\{w \neq 0\} \rightarrow\left(\mathcal{Z}_{P} / \operatorname{ker} \lambda_{A^{\prime}}^{S}\right) \cap\{w \neq 0\}$.

Proof. As is easily checked, $f$ preserves $\mathcal{Z}_{P}$ (see (7.2) ) and is a diffeomorphism, so it suffices to show that $f$ is weakly equivariant with respect to the actions of $\operatorname{ker} \lambda_{A}^{S}$ and $\operatorname{ker} \lambda_{A^{\prime}}^{S}$.

Remember the description of $\operatorname{ker} \lambda_{A}^{S}$ in (7.8). Since the complex conjugate of an element in $S^{1}$ is equal to its inverse and $|h|=1$ for $h \in S^{1}$, we have

$$
\begin{aligned}
f\left(\boldsymbol{h}^{\mathbf{u}_{1}} h^{b_{1}} z_{1}, \ldots, \boldsymbol{h}^{\mathbf{u}_{n}} h^{b_{n}} z_{n}, h_{1} w_{1}, \ldots, h_{n} w_{n}, h w\right) \\
=\left(\boldsymbol{h}^{\mathbf{u}_{1}} h^{b_{1}} z_{1}, \ldots, \boldsymbol{h}^{\mathbf{u}_{i-1}} h^{b_{i-1}} z_{i-1},\left(\frac{h w}{|w|}\right) \boldsymbol{h}^{-\mathbf{u}_{i}} h^{-b_{i}} \bar{z}_{i},\right. \\
\quad\left(\frac{h w}{|w|}\right)^{2} \boldsymbol{h}^{-\mathbf{u}_{i+1}} h^{-b_{i+1}} \bar{z}_{i+1}, \ldots,\left(\frac{h w}{|w|}\right)^{2} \boldsymbol{h}^{-\mathbf{u}_{j-1}} h^{-b_{j-1}} \bar{z}_{j-1}, \\
\quad\left(\frac{h w}{|w|}\right) \boldsymbol{h}^{\mathbf{u}_{j}} h^{b_{j}} z_{j}, \boldsymbol{h}^{\mathbf{u}_{j+1}} h^{b_{j+1}} z_{j+1}, \ldots, \boldsymbol{h}^{\mathbf{u}_{n}} h^{b_{n}} z_{n}, \\
\left.\quad h_{1} w_{1}, \ldots, h_{i-1} w_{i-1}, h_{i}^{-1} \bar{w}_{i}, \ldots, h_{j-1}^{-1} \bar{w}_{j-1}, h_{j} w_{j}, \ldots, h_{n} w_{n}, h w\right) .
\end{aligned}
$$


Here, since $\mathbf{u}_{k}=\left[0, \ldots, 0, a_{k}, 1,0 \ldots, 0\right]$, it follows from 7.13 that we have

$$
\begin{aligned}
& \boldsymbol{h}^{-\mathbf{u}_{i}}=h_{i-1}^{-a_{i}} h_{i}^{-1}=h_{i-1} h_{i}^{-1}, \\
& \boldsymbol{h}^{-\mathbf{u}_{k}}=h_{k-1}^{-a_{k}} h_{k}^{-1}=h_{k-1}^{-1} h_{k}^{-1} \quad(i+1 \leq k \leq j-1), \\
& \boldsymbol{h}^{\mathbf{u}_{j}}=h_{j-1}^{a_{j}} h_{j}=h_{j-1}^{-1} h_{j},
\end{aligned}
$$

and note that $\boldsymbol{h}^{\mathbf{u}_{k}}$ does not contain $h_{i}, h_{i+1}, \ldots, h_{j-1}$ unless $i \leq k \leq j$. On the other hand, it follows from the definition of $a_{k}^{\prime}$ 's in (7.14) that we have

$$
\begin{aligned}
& \mathbf{u}_{k}^{\prime}=\mathbf{u}_{k}, b_{k}^{\prime}=b_{k} \quad \text { for } k \neq i, j, \\
& b_{k}^{\prime}=b_{k}=1 \quad \text { for } i+1 \leq k \leq j-1, \\
& b_{i}^{\prime}=b_{j}^{\prime}=1, \quad b_{i}=b_{j}=0 .
\end{aligned}
$$

Hence, the right hand side of (7.15) is written as

$$
\begin{aligned}
& \left(\boldsymbol{h}^{\mathbf{u}_{1}^{\prime}} h^{b_{1}^{\prime}} z_{1}, \ldots, \boldsymbol{h}^{\mathbf{u}_{i-1}^{\prime}} h^{b_{i-1}^{\prime}} z_{i-1},\left(\frac{w}{|w|}\right) h_{i-1} h_{i}^{-1} h^{b_{i}^{\prime}} \bar{z}_{i},\right. \\
& \quad\left(\frac{w}{|w|}\right)^{2} h_{i}^{-1} h_{i+1}^{-1} h^{b_{i+1}^{\prime}} \bar{z}_{i+1}, \ldots,\left(\frac{w}{|w|}\right)^{2} h_{j-2}^{-1} h_{j-1}^{-1} h^{b_{j-1}^{\prime}} \bar{z}_{j-1}, \\
& \quad\left(\frac{w}{|w|}\right) h_{j-1}^{-1} h_{j} h^{b_{j}^{\prime}} z_{j}, \boldsymbol{h}^{\mathbf{u}_{j+1}^{\prime}} h^{b_{j+1}^{\prime}} z_{j+1}, \ldots, \boldsymbol{h}^{\mathbf{u}_{n}^{\prime}} h^{b_{n}^{\prime}} z_{n}, \\
& \left.\quad h_{1} w_{1}, \ldots, h_{i-1} w_{i-1}, h_{i}^{-1} \bar{w}_{i}, \ldots, h_{j-1}^{-1} \bar{w}_{j-1}, h_{j} w_{j}, \ldots, h_{n} w_{n}, h w\right) .
\end{aligned}
$$

We set $\boldsymbol{h}^{\prime}=\left(h_{1}, \ldots, h_{i-1}, h_{i}^{-1}, \ldots, h_{j-1}^{-1}, h_{j}, \ldots, h_{n}, h\right)$. Then

$$
\begin{aligned}
& \boldsymbol{h}^{\mathbf{u}_{k}^{\prime}}=\left(\boldsymbol{h}^{\prime}\right)^{\mathbf{u}_{k}^{\prime}} \quad(\text { unless } i \leq k \leq j), \\
& h_{i-1} h_{i}^{-1}=\left(\boldsymbol{h}^{\prime}\right)^{\mathbf{u}_{i}^{\prime}}, \quad h_{k}^{-1} h_{k+1}^{-1}=\left(\boldsymbol{h}^{\prime}\right)^{\mathbf{u}_{k}^{\prime}} \quad(i+1 \leq k \leq j-1), \quad h_{j-1}^{-1} h_{j}=\left(\boldsymbol{h}^{\prime}\right)^{\mathbf{u}_{j}^{\prime}} .
\end{aligned}
$$

This together with (7.15) and (7.16) shows that $f$ is $\theta$-equivariant with respect to the actions of $\operatorname{ker} \lambda_{A}^{S}$ and $\operatorname{ker} \lambda_{A^{\prime}}^{S}$, where $\theta$ is the automorphism of $\left(S^{1}\right)^{2 n+1}$ which maps $\boldsymbol{h}$ to $\boldsymbol{h}^{\prime}$, proving the lemma.

Now we are ready to prove Proposition 7.1 .

Proof of Proposition 7.1. Let $\hat{\varphi}_{A}$ and $\hat{f}$ be the diffeomorphisms in Lemmas 7.4 and 7.5, respectively. We consider the composition

$$
\hat{\varphi}_{A^{\prime}} \circ \hat{f} \circ \hat{\varphi}_{A}^{-1}: L \cap\{u \neq 0\} \rightarrow L \cap\{u \neq 0\} .
$$

Setting $s_{i}=\sqrt{1-\left|v_{i}\right|^{2}}$, we can see that

$$
\begin{aligned}
& \left(\hat{\varphi}_{A^{\prime}} \circ \hat{f} \circ \hat{\varphi}_{A}^{-1}\right)\left(\left[v_{1}, \ldots, v_{n}, u\right]\right) \\
& =\left(\hat{\varphi}_{A^{\prime}} \circ \hat{f}\right)\left(\left[s_{1}, \ldots, s_{n}, v_{1}, \ldots, v_{n}, u\right]\right) \\
& =\hat{\varphi}_{A^{\prime}}\left(\left[s_{1}, \ldots, s_{i-1}, s_{i} \frac{u}{|u|}, s_{i+1}\left(\frac{u}{|u|}\right)^{2}, \ldots, s_{j-1}\left(\frac{u}{|u|}\right)^{2}, s_{j} \frac{u}{|u|}, s_{j+1}, \ldots, s_{n},\right.\right. \\
& \left.\left.\quad v_{1}, \ldots, v_{i-1}, \bar{v}_{i}, \ldots, \bar{v}_{j-1}, v_{j}, \ldots, v_{n}, u\right]\right) \\
& =\left[f_{1} v_{1}, \ldots, f_{i-1} v_{i-1}, f_{i} \bar{v}_{i}, \ldots, f_{j-1} \bar{v}_{j-1}, f_{j} v_{j}, \ldots, f_{n} v_{n}, f_{0} u\right]
\end{aligned}
$$

where each $f_{i}$ is a Laurent monomial of $u /|u|$ with coefficient 1 by (7.10). Since the coordinates in (7.17) are homogeneous with respect to the $S^{1}$-action on $\tilde{L}$ defined 
in (7.9) and $f_{0} \in S^{1}$, we may assume $f_{0}=1$. Moreover, the weights of $u$ and $v_{i}$ with respect to the $S^{1}$-action are -2 and 1 respectively from (7.9), the last term in (7.17) must be equal to

$$
\left[v_{1}, \ldots, v_{i-1},\left(\frac{\bar{u}}{|u|}\right) \bar{v}_{i}, \ldots,\left(\frac{\bar{u}}{|u|}\right) \bar{v}_{j-1}, v_{j}, \ldots, v_{n}, u\right]
$$

where $\bar{u} /|u|=(u /|u|)^{-1}$.

Note that $L \cap\{|u|=r\}$ for $0<r<1 / \sqrt{2}$ is diffeomorphic to $\mathbb{R} P^{2 n-1}$. In fact, if we write $v_{i}=x_{i}+\sqrt{-1} y_{i}$, then a diffeomorphism is given by

$$
\begin{aligned}
L \cap\{|u|=r\} & \rightarrow \mathbb{R} P^{2 n-1} \\
{\left[v_{1}, \ldots, v_{n}, r\right] } & \mapsto\left[x_{1}, \ldots, x_{n}, y_{1}, \ldots, y_{n}\right] .
\end{aligned}
$$

Then $\hat{\varphi_{A^{\prime}}} \circ \hat{f} \circ \hat{\varphi}_{A}^{-1}$ restricted to $|u|=r$ is a self-diffeomorphism $\psi$ of $\mathbb{R} P^{2 n-1}$ which maps $\left[x_{1}, \ldots, x_{n}, y_{1}, \ldots, y_{n}\right]$ to

$$
\left[x_{1}, \ldots, x_{n}, y_{1}, \ldots, y_{i-1},-y_{i}, \ldots,-y_{j-1}, y_{j}, \ldots, y_{n}\right] \in \mathbb{R} P^{2 n-1} .
$$

Note that $\psi$ is independent of the choice of $r$. Since $j-i$ is even, $\psi$ is isotopic to the identity map on $\mathbb{R} P^{2 n-1}$. Therefore $\hat{\varphi}_{A^{\prime}} \circ \hat{f} \circ \hat{\varphi}_{A}^{-1}$ restricted to $L \cap\{|u| \geq 1 / 2 \sqrt{2}\}$ extends to a self-diffeomorphism of $L$ as the identity map on a neighborhood of $L \cap\{u=0\}$. This means that $\hat{f}$ restricted to $\left(\mathcal{Z}_{P} / \operatorname{ker} \lambda_{A}^{S}\right) \cap\{|w| \geq 1 / 2 \sqrt{2}\}$ extends to a diffeomorphism $\mathcal{Z}_{P} / \operatorname{ker} \lambda_{A}^{S} \rightarrow \mathcal{Z}_{P} / \operatorname{ker} \lambda_{A^{\prime}}^{S}$

\section{Projectivity}

It is known that every toric manifold of complex dimension less than 3 is projective. However, in general, there are many non-projective toric manifolds. Oda's 3 -fold, which is of Type 2 in our terminology, is known as the simplest non-projective toric manifold. In this section, we will check the projectivity of a toric manifold associated with a fan over $\mathcal{C}_{n}$. It turns out that our toric manifold is projective unless it is of Type 2 and only one toric manifold is projective in Type 2 in each dimension $\geq 3$ while the others in Type 2 are non-projective (Propositions 8.1 and 8.3 ).

First we discuss the toric manifolds of Type 2. Recall that the matrix $A$ of Type 2 is of the form:

$$
\left[\begin{array}{ccccc}
1 & 0 & \ldots & 0 & a_{1} \\
a_{2} & 1 & \ldots & 0 & 0 \\
\vdots & \vdots & \ddots & \vdots & \vdots \\
0 & 0 & \ldots & 1 & 0 \\
0 & 0 & \ldots & a_{n} & 1
\end{array}\right]
$$

where $a_{i}= \pm 1$ and the number of $a_{i}$ 's equal to 1 is odd.

Proposition 8.1. Toric manifolds of Type 2 are non-projective if there are at least three 1 's in $\left\{a_{1}, \ldots, a_{n}\right\}$.

Proof. Since the matrix $A$ is determined by the ordered set $\left(a_{1}, \ldots, a_{n}\right)$, we express $A$ as $\left(a_{1}, \ldots, a_{n}\right)$. By projecting the fan $\Delta$ associated with $A=\left(a_{1}, \ldots, a_{n}\right)$ onto $\mathbb{Z}^{n} /\left(\mathbf{a}_{k}\right)$, we obtain the fan $\Delta_{k}$ associated with $A_{k}=\left(a_{1}, \ldots,-a_{k} a_{k+1}, \ldots, a_{n}\right)$ 4 Note that the toric manifold $X\left(\Delta_{k}\right)$ is an invariant subvariety of the toric manifold $X(\Delta)$. Furthermore, if $a_{k}=-1$, then the ordered set $A_{k}$ has one less $(-1)$ 's than

\footnotetext{
${ }^{4}$ This is similar to the argument in the proof of Proposition 3.2
} 
the ordered set $A$. We repeat this process until we get the ordered set $A_{*}$ all of whose elements are equal to 1 . Since $A$ has at least three 1's from the hypothesis, the size of $A_{*}$ is at least three. If $A_{*}$ has exactly three 1 's, then the toric manifold associated with $A_{*}$ is Oda's 3 -fold. Since every invariant subvariety of a projective toric variety is also projective, $X(\Delta)$ is non-projective. If $A_{*}$ has at least five 1's, then the new ordered set $A^{\prime}$ obtained from the projection as above has two less 1's compared with $A_{*}$. Hence, by repeating projections suitably, we get the ordered set consisting of three 1's. Hence, if the ordered set $A$ has at least three 1's, then the corresponding toric manifold is non-projective.

The following proposition provides a criterion of whether a toric manifold is projective or not.

Proposition 8.2. [10, page 70] A toric manifold $X$ of complex dimension $n$ is projective if and only if there is a continuous piecewise linear function $\psi$ on the support $\left|\Delta_{X}\right|=\mathbb{R}^{n}$ of the fan $\Delta_{X}$ associated with $X$ that satisfies the following two conditions:

(C1) The restriction of $\psi$ to each $n$-dimensional cone $\sigma$, denoted by $\psi_{\sigma}$, is linear.

(C2) $\psi_{\sigma}$ satisfies $\psi_{\sigma}\left(\mathbf{v}_{i}\right)>\psi\left(\mathbf{v}_{i}\right)$ for $\mathbf{v}_{i} \notin \sigma$,

where $\mathbf{v}_{i}$ 's are the primitive edge vectors in the fan $\Delta_{X}$.

We think of $\mathbf{v}_{i}$ 's as column vectors as before. Since $\Delta_{X}$ is nonsingular, $Q=$ $\left[\mathbf{v}_{i}\right]_{\mathbf{v}_{i} \in \sigma}$ is a unimodular matrix and we denote by $\mathbf{u}_{i}$ the row vector of $Q^{-1}$ corresponding to $\mathbf{v}_{i}$, i.e., $\mathbf{u}_{i} \mathbf{v}_{j}=\delta_{i j}$. Then $\psi_{\sigma}$ is of the form

$$
\psi_{\sigma}=\sum_{\mathbf{v}_{i} \in \sigma} \psi\left(\mathbf{v}_{i}\right) \mathbf{u}_{i} .
$$

Proposition 8.3. Each toric manifold $X(A, \mathbf{b})$ is projective except when the toric manifold is of Type 2 such that the number of $a_{i}$ 's equal to 1 is more than one.

Proof. It is well-known that every Bott manifold is a projective toric manifold. Since a toric manifold of Type 1 is a blow-up of a Bott manifold, it is also projective. For the other types, we will find a continuous piecewise linear function $\psi$ on $\mathbb{R}^{n}$ satisfying $(\mathrm{C} 1)$ and $(\mathrm{C} 2)$ in Proposition 8.2

Remember that the minimal non-faces of $\mathcal{C}_{\mathrm{n}}$ are

$$
\{i, n+1\},\{i, 2 n+1\} \text { for } 1 \leq i \leq n \text { and }\{n+1, \ldots, 2 n\} .
$$

We may take $\mathbf{v}_{1}=-\mathbf{e}_{1}, \ldots, \mathbf{v}_{n}=-\mathbf{e}_{n}$, so that $\mathbf{v}_{n+1}=\mathbf{a}_{1}, \ldots, \mathbf{v}_{2 n}=\mathbf{a}_{n}$ and $\mathbf{v}_{2 n+1}=\mathbf{b}$.

We first deal with the toric manifolds of Type 0 .

Type 0. Recall that

$$
\left[\mathbf{a}_{1}, \ldots, \mathbf{a}_{n}\right]=\left[\begin{array}{ccccc}
1 & 0 & \ldots & 0 & -1 \\
-1 & 1 & \ldots & 0 & 0 \\
\vdots & \vdots & \ddots & \vdots & \vdots \\
0 & 0 & \ldots & 1 & 0 \\
0 & 0 & \ldots & -1 & 1
\end{array}\right], \quad \mathbf{b}=\left[\begin{array}{c}
b_{1} \\
b_{2} \\
\vdots \\
\vdots \\
b_{n}
\end{array}\right] \quad \text { with } \sum_{i=1}^{n} b_{i}=1
$$


Let $b:=\max \left\{\left|b_{1}\right|, \ldots,\left|b_{n}\right|\right\}$, and let $\psi$ be the piecewise linear function defined by

$$
\psi\left(-\mathbf{e}_{i}\right)=\psi\left(\mathbf{a}_{i}\right)=-1 \text { for } 1 \leq i \leq n, \text { and } \psi(\mathbf{b})=-\frac{n(n-1)}{2} b .
$$

We shall show that our $\psi$ satisfies (C2). We divide the proof into two cases according to whether the $n$-dimensional cone $\sigma$ contains $\mathbf{b}$ or not.

Case 1: $\mathbf{b} \notin \sigma$. In this case, $\sigma$ contains some $-\mathbf{e}_{i}$ as an edge vector. Through a cyclic permutation on $[n]$, we may assume that $\sigma$ contains $-\mathbf{e}_{n}$. Then the matrix $Q=\left[\mathbf{v}_{i}\right]_{\mathbf{v}_{i} \in \sigma}$ is equal to $-E_{n}$ or it is of the form

$$
\begin{aligned}
Q= & {\left[\mathbf{a}_{1}, \ldots, \mathbf{a}_{r_{1}},-\mathbf{e}_{r_{1}+1}, \ldots,-\mathbf{e}_{r_{1}+s_{1}}, \ldots,\right.} \\
& \left.\mathbf{a}_{m+1}, \ldots, \mathbf{a}_{m+r_{q}},-\mathbf{e}_{m+r_{q}+1}, \ldots,-\mathbf{e}_{m+r_{q}+s_{q}}\right],
\end{aligned}
$$

where $m=\sum_{i=1}^{q-1}\left(r_{i}+s_{i}\right), m+r_{q}+s_{q}=n, r_{1} \geq 0, r_{i} \geq 1$ for $i \geq 2$, and $s_{i} \geq 1$ for $i \geq 1$.

If $Q=-E_{n}$, then $Q^{-1}=-E_{n}$ and $\psi_{\sigma}=[1, \ldots, 1]$ by 8.1 and 8.2). Hence, $\psi_{\sigma}\left(\mathbf{a}_{i}\right)=0>\psi\left(\mathbf{a}_{i}\right)$ for $1 \leq i \leq n$ and $\psi_{\sigma}(\mathbf{b})=1>\psi(\mathbf{b})$ by 8.2).

If $Q \neq-E_{n}$, then $Q^{-1}$ is as follows:

$$
Q^{-1}=\left[\begin{array}{lll}
D_{r_{1}, s_{1}} & & \\
& \ddots & \\
& & D_{r_{q}, s_{q}}
\end{array}\right]
$$

where

$$
\left.D_{r, s}=\left[\begin{array}{ccc|ccc}
1 & & & & & \\
\vdots & \ddots & & & & \\
1 & \ldots & 1 & & & \\
\hline-1 & \ldots & -1 & -1 & & \\
& & & & \ddots & \\
& & & & & -1
\end{array}\right]\right\} s
$$

Therefore, it follows from (8.1) and (8.2) that $\psi_{\sigma}$ is given by

$$
\begin{aligned}
\psi_{\sigma}=[ & -1, \ldots,-1] Q^{-1} \\
= & -\left(r_{1}-1\right),-\left(r_{1}-2\right), \ldots,-1,0,1, \ldots, 1, \\
& \ldots \ldots \ldots \ldots \ldots \ldots \ldots \\
& \left.\quad-\left(r_{q}-1\right),-\left(r_{q}-2\right), \ldots,-1,0,1, \ldots, 1\right],
\end{aligned}
$$

where 0's correspond to $\mathbf{a}_{r_{1}}, \mathbf{a}_{r_{1}+s_{1}+r_{2}}, \ldots, \mathbf{a}_{m+r_{q}}$ in this order. Then the vectors $-\mathbf{e}_{i}$ and $\mathbf{a}_{i}$ not contained in $\sigma$ satisfy

$$
\begin{aligned}
& 0 \leq \psi_{\sigma}\left(-\mathbf{e}_{i}\right) \leq r_{j}-1 \text { for some } j, \\
& \psi_{\sigma}\left(\mathbf{a}_{i}\right)=0 \text { or } r_{j} \text { for some } j .
\end{aligned}
$$

This together with (8.2) shows that (C2) is satisfied for those $-\mathbf{e}_{i}$ and $\mathbf{a}_{i}$ not contained in $\sigma$. It also follows from (8.2) and (8.5) that

$$
\psi_{\sigma}(\mathbf{b}) \geq-\left(\sum_{k=1}^{q} \frac{r_{k}\left(r_{k}-1\right)}{2}+\sum_{k=1}^{q} s_{k}\right) b>-\frac{n(n-1)}{2} b=\psi(\mathbf{b}) .
$$

Hence, our $\psi$ satisfies (C1) and (C2). 
Case 2: $\mathbf{b} \in \sigma$. In this case none of $-\mathbf{e}_{i}$ 's are contained in $\sigma$ and some $\mathbf{a}_{i}$ is not contained in $\sigma$. Similarly to Case 1, we may assume that $\mathbf{a}_{n}$ is not contained in $\sigma$. Then

$$
Q=\left[\mathbf{a}_{1}, \ldots, \mathbf{a}_{n-1}, \mathbf{b}\right]=\left[\begin{array}{ccccc}
1 & & & & b_{1} \\
-1 & 1 & & & b_{2} \\
& \ddots & \ddots & & \vdots \\
& & -1 & 1 & b_{n-1} \\
& & & -1 & b_{n}
\end{array}\right]
$$

and hence

$$
Q^{-1}=\left[\begin{array}{ccccc}
\sum_{k=2}^{n} b_{k} & -b_{1} & \ldots & -b_{1} & -b_{1} \\
\sum_{k=3}^{n} b_{k} & \sum_{k=3}^{n} b_{k} & \ldots & -b_{1}-b_{2} & -b_{1}-b_{2} \\
\vdots & \vdots & \ddots & \vdots & \vdots \\
b_{n} & b_{n} & \ldots & b_{n} & -\sum_{k=1}^{n-1} b_{k} \\
1 & 1 & \cdots & 1 & 1
\end{array}\right]
$$

The $i$ th component of $\psi_{\sigma}=\left[-1, \ldots,-1,-\frac{n(n-1)}{2} b\right] Q^{-1}$ is equal to

$$
\sum_{\ell=1}^{i-1}\left(\sum_{k=1}^{\ell} b_{k}\right)-\sum_{\ell=i+1}^{n}\left(\sum_{k=\ell}^{n} b_{k}\right)-\frac{n(n-1)}{2} b .
$$

The edge vectors not contained in $\sigma$ are $-\mathbf{e}_{1}, \ldots,-\mathbf{e}_{n}$ and $\mathbf{a}_{n}$, and it follows from (8.2) and (8.7) that they satisfy

$$
\begin{aligned}
\psi_{\sigma}\left(-\mathbf{e}_{i}\right) & =-\sum_{\ell=1}^{i-1}\left(\sum_{k=1}^{\ell} b_{k}\right)+\sum_{\ell=i+1}^{n}\left(\sum_{k=\ell}^{n} b_{k}\right)+\frac{n(n-1)}{2} b>-1=\psi\left(-\mathbf{e}_{i}\right) \\
\psi_{\sigma}\left(\mathbf{a}_{n}\right) & =\sum_{\ell=2}^{n}\left(\sum_{k=\ell}^{n} b_{k}\right)+\sum_{\ell=1}^{n-1}\left(\sum_{k=1}^{\ell} b_{k}\right)=(n-1) \sum_{k=1}^{n} b_{k}=n-1>-1=\psi\left(\mathbf{a}_{n}\right) .
\end{aligned}
$$

Hence, our $\psi$ satisfies $(\mathrm{C} 1)$ and $(\mathrm{C} 2)$.

Type 2 having only one $a_{i}$ equal to 1 and Type 3 . Recall that, in Type 3 ,

$$
\left[\mathbf{a}_{1}, \ldots, \mathbf{a}_{n}\right]=\left[\begin{array}{ccccc}
1 & 0 & \ldots & 0 & a \\
-1 & 1 & \ldots & 0 & 0 \\
\vdots & \vdots & \ddots & \vdots & \vdots \\
0 & 0 & \ldots & 1 & 0 \\
0 & 0 & \ldots & -1 & 1
\end{array}\right], \quad \mathbf{b}=\frac{1}{\operatorname{det} A} \sum_{i=1}^{n} \mathbf{a}_{i}=\left[\begin{array}{c}
1 \\
0 \\
\vdots \\
\vdots \\
0
\end{array}\right]
$$

If $a=1$ in 8.8), then $\left[\mathbf{a}_{1}, \ldots, \mathbf{a}_{n}, \mathbf{b}\right]$ is of Type 2 such that the number of $a_{i}$ 's equal to 1 is exactly one. Hence, it remains to prove that the toric manifold associated with $\left[\mathbf{a}_{1}, \ldots, \mathbf{a}_{n}, \mathbf{b}\right]$ in 8.8 is projective when $a \neq 0,-1$.

Let $\psi$ be the piecewise linear function defined by

$$
\begin{aligned}
& \psi\left(-\mathbf{e}_{i}\right)=-1 \text { for } 1 \leq i \leq n, \quad \psi\left(\mathbf{a}_{i}\right)=-1 \text { for } 1 \leq i \leq n-1 \\
& \psi\left(\mathbf{a}_{n}\right)=-n|a|, \quad \psi(\mathbf{b})=-(n-1) .
\end{aligned}
$$

To show that $\psi$ satisfies (C2), we divide the proof into four cases according to whether $\sigma$ contains the edge vectors $\mathbf{a}_{n}$ and $\mathbf{b}$. 
Case 1: $\mathbf{b} \notin \sigma$ and $\mathbf{a}_{n} \notin \sigma$. In this case, $-\mathbf{e}_{n} \in \sigma$ and the matrix $Q$ is either $-E_{n}$ or of the form in (8.3). If $Q=-E_{n}$, then $Q^{-1}=-E_{n}$ and $\psi_{\sigma}=[1, \ldots, 1]$, and hence

$$
\begin{aligned}
& \psi_{\sigma}\left(\mathbf{a}_{i}\right)=0>\psi\left(\mathbf{a}_{i}\right) \quad(1 \leq i<n), \\
& \psi_{\sigma}\left(\mathbf{a}_{n}\right)=a+1>\psi\left(\mathbf{a}_{n}\right), \text { and } \\
& \psi_{\sigma}(\mathbf{b})=1>-(n-1) .
\end{aligned}
$$

Hence, (C2) is satisfied. If $Q \neq-E_{n}$, then $\psi_{\sigma}$ is the same as (8.5). Thus (C2) is satisfied for the edge vectors not contained in $\sigma$ except for $\mathbf{a}_{n}$ and $\mathbf{b}$, see (8.6). As for $\mathbf{a}_{n}$ and $\mathbf{b}$, since $\psi_{\sigma}\left(\mathbf{a}_{n}\right)=1-a\left(r_{1}-1\right)$ and $r_{1} \leq n-1$, we get

$$
\psi_{\sigma}\left(\mathbf{a}_{n}\right)>-(n-2)|a| \geq-n|a|=\psi\left(\mathbf{a}_{n}\right),
$$

and

$$
\psi_{\sigma}(\mathbf{b})=-\left(r_{1}-1\right) \geq-(n-2)>-(n-1)=\psi(\mathbf{b}) .
$$

Hence, our $\psi$ satisfies $(\mathrm{C} 1)$ and $(\mathrm{C} 2)$.

Case 2: $\mathbf{b} \notin \sigma$ and $\mathbf{a}_{n} \in \sigma$. In this case, the matrix $Q$ is obtained from the matrix in (8.3) by replacing $-\mathbf{e}_{n}$ with $\mathbf{a}_{n}$, that is,

$$
\begin{aligned}
Q=\left[\mathbf{a}_{1}, \ldots, \mathbf{a}_{r_{1}},-\mathbf{e}_{r_{1}+1}, \ldots,-\mathbf{e}_{r_{1}+s_{1}}, \ldots,\right. \\
\left.\quad \mathbf{a}_{m+1}, \ldots, \mathbf{a}_{m+r_{q}},-\mathbf{e}_{m+r_{q}+1}, \ldots,-\mathbf{e}_{m+r_{q}+s_{q}-1}, \mathbf{a}_{n}\right] .
\end{aligned}
$$

We consider two subcases depending on whether $-\mathbf{e}_{n-1} \in \sigma$ or not.

Subcase 2-1: $-\mathbf{e}_{n-1} \in \sigma$. In this case, $m+r_{q}<n-1$ and we have

$$
Q^{-1}=\left[\begin{array}{cccc|c}
D_{r_{1}, s_{1}} & & & & \\
& \ddots & & \\
& & D_{r_{q-1}, s_{q-1}} & & \\
& & & D_{r_{q}, s_{q}-1} & \\
\hline & & & & 1
\end{array}\right],
$$

where $D_{r, s}$ is the matrix $(8.4)$ and $\overline{\mathbf{a}}=[\underbrace{-a, \ldots,-a}_{r_{1}}, a, 0, \ldots, 0]^{T}$. Then

$$
\begin{aligned}
\psi_{\sigma}= & {[-1, \ldots,-1,-n|a|] Q^{-1} } \\
= & {\left[-\left(r_{1}-1\right),-\left(r_{1}-2\right), \ldots,-1,0,1 \ldots, 1\right.} \\
& \ldots \ldots \ldots \ldots \ldots \ldots \ldots,-1,0,1 \ldots, 1 \\
& \left.-\left(r_{q-1}-1\right),-\left(r_{q-1}-2\right), \ldots,-1, \ldots, 1, \ldots, 1,\left(r_{1}-1\right) a-n|a|\right] . \\
& -\left(r_{q}-1\right),-\left(r_{q}-2\right), \ldots,-1,0,1, \ldots
\end{aligned}
$$

Note that $\psi_{\sigma}$ is the same as (8.5) except the last coordinate. Hence, 8.6) shows that $\psi_{\sigma}$ satisfies (C2) for $-\mathbf{e}_{i} \notin \sigma$ except for $-\mathbf{e}_{n}$. The function $\psi_{\sigma}$ also satisfies (C2) for $\mathbf{a}_{i} \notin \sigma$ except for $\mathbf{a}_{n-1}$. On the other hand, since $r_{1} \leq n-2$, we can see that

$$
\begin{aligned}
& \psi_{\sigma}\left(-\mathbf{e}_{n}\right)=n|a|-\left(r_{1}-1\right) a>-1=\psi\left(-\mathbf{e}_{n}\right), \\
& \psi_{\sigma}\left(\mathbf{a}_{n-1}\right)=1+n|a|-\left(r_{1}-1\right) a>-1=\psi\left(\mathbf{a}_{n-1}\right), \text { and } \\
& \psi_{\sigma}(\mathbf{b})=-\left(r_{1}-1\right)>-(n-1)=\psi(\mathbf{b}) .
\end{aligned}
$$


This proves that $\psi_{\sigma}$ satisfies (C2).

Subcase 2-2: $-\mathbf{e}_{n-1} \notin \sigma$ In this case, $m+r_{q}=n-1$ and we have

$$
Q^{-1}=\left[\begin{array}{llll}
D_{r_{1}, s_{1}} & & & \bar{A} \\
& \ddots & & \\
& & D_{r_{q-1}, s_{q-1}} & \\
& & & D
\end{array}\right],
$$

where $\bar{A}$ is the $\left(r_{1}+1\right) \times\left(r_{q}+1\right)$ matrix and $D$ is the square matrix of size $\left(r_{q}+1\right)$ such that

$$
\bar{A}=\left[\begin{array}{ccc}
-a & \ldots & -a \\
\vdots & \ldots & \vdots \\
-a & \ldots & -a \\
a & \ldots & a
\end{array}\right] \text { and } D=\left[\begin{array}{cccc}
1 & & & \\
1 & 1 & & \\
\vdots & \vdots & \ddots & \\
1 & 1 & \ldots & 1
\end{array}\right]
$$

Then

$$
\begin{aligned}
& \psi_{\sigma}=[-1, \ldots,-1,-n|a|] Q^{-1} \\
& =\left[-\left(r_{1}-1\right),-\left(r_{1}-2\right), \ldots,-1,0,1 \ldots, 1,\right. \\
& -\left(r_{q-1}-1\right),-\left(r_{q-1}-2\right), \ldots,-1,0,1 \ldots, 1, \\
& \left.\left(r_{1}-1\right) a-r_{q}-n|a|, \ldots,\left(r_{1}-1\right) a-n|a|\right] .
\end{aligned}
$$

Since $\psi_{\sigma}$ is the same as (8.5) except for the coordinates from $m+1$ to $n$, it is enough to check (C2) for $-\mathbf{e}_{i} \notin \sigma(m+1 \leq i \leq n), \mathbf{a}_{m}$, and $\mathbf{b}$. Note that $n=m+r_{q}+1$. Since $r_{1} \leq n-2$, we can see that

$$
\begin{aligned}
& \psi_{\sigma}\left(-\mathbf{e}_{i}\right)=n|a|+(n-i)-\left(r_{1}-1\right) a>-1=\psi\left(-\mathbf{e}_{i}\right) \quad(m+1 \leq i \leq n), \\
& \psi_{\sigma}\left(\mathbf{a}_{m}\right)=1+n|a|+r_{q}-\left(r_{1}-1\right) a>-1=\psi\left(\mathbf{a}_{m}\right), \\
& \psi_{\sigma}(\mathbf{b})=-\left(r_{1}-1\right)>-(n-1)=\psi(\mathbf{b}) .
\end{aligned}
$$

Hence, our $\psi$ satisfies (C1) and (C2).

Case $3: \mathbf{b} \in \sigma$ and $\mathbf{a}_{n} \notin \sigma$. Since $Q=\left[\mathbf{a}_{1}, \ldots, \mathbf{a}_{n-1}, \mathbf{b}\right]$, we have

$$
Q^{-1}=\left[\begin{array}{cccc}
0 & -1 & \ldots & -1 \\
\vdots & \ddots & \ddots & \vdots \\
0 & \cdots & 0 & -1 \\
1 & \cdots & 1 & 1
\end{array}\right]
$$

Hence

$$
\psi_{\sigma}=[-1, \ldots,-1,-(n-1)] Q^{-1}=[-(n-1),-(n-2), \ldots,-1,0] .
$$

The vectors $-\mathbf{e}_{i}$ for $1 \leq i \leq n$ and $\mathbf{a}_{n}$ are not contained in $\sigma$, and we can see that

$$
\begin{aligned}
& \psi_{\sigma}\left(-\mathbf{e}_{i}\right)=n-i>-1=\psi\left(-\mathbf{e}_{i}\right), \\
& \psi_{\sigma}\left(\mathbf{a}_{n}\right)=-(n-1) a>-n|a|=\psi\left(\mathbf{a}_{n}\right),
\end{aligned}
$$

since $a \neq 0$. Thus, $\psi_{\sigma}$ satisfies (C2). 
Case 4: $\mathbf{b} \in \sigma$ and $\mathbf{a}_{n} \in \sigma$. Since the matrix $Q=\left[\mathbf{a}_{1}, \ldots, \mathbf{a}_{k-1}, \mathbf{b}, \mathbf{a}_{k+1}, \ldots, \mathbf{a}_{n}\right]$ for some $1 \leq k \leq n-1$, we obtain

$$
Q^{-1}=\left[\begin{array}{ccccccc}
0 & -1 & \ldots & -1 & & & \\
\vdots & \ddots & \ddots & \vdots & & & \\
0 & \ldots & 0 & -1 & & & \\
1 & \ldots & 1 & 1 & -a & \ldots & -a \\
& & & & 1 & & \\
& & & & \vdots & \ddots & \\
& & & & 1 & \ldots & 1
\end{array}\right],
$$

where the number of 0 's on the main diagonal is $k-1$. Hence

$$
\begin{aligned}
\psi_{\sigma}= & {[-1, \ldots,-1,-(n-1),-1, \ldots,-1,-n|a|] Q^{-1} } \\
= & {[-(n-1), \ldots,-(n-1)+k-2,-(n-1)+k-1,} \\
& (n-1) a-(n-k-1)-n|a|, \ldots,(n-1) a-n|a|] .
\end{aligned}
$$

The edge vectors not contained in $\sigma$ are $\mathbf{a}_{k}$ and $-\mathbf{e}_{i}$ for $i=1 \ldots, n$, and we can see that

$$
\begin{aligned}
& \psi_{\sigma}\left(-\mathbf{e}_{i}\right)= \begin{cases}n-i>-1=\psi\left(-\mathbf{e}_{i}\right) & (1 \leq i \leq k), \\
-(n-1) a+(n-i)+n|a|>-1=\psi\left(-\mathbf{e}_{i}\right) & (k+1 \leq i \leq n),\end{cases} \\
& \psi_{\sigma}\left(\mathbf{a}_{k}\right)=-(n-1) a-1+n|a|>-1=\psi\left(\mathbf{a}_{k}\right),
\end{aligned}
$$

since $a \neq 0$. Therefore, $\psi_{\sigma}$ satisfies (C2).

This completes the proof of the proposition.

\section{APPENDIX .}

In this section, we provide the proof of Proposition 7.2 and give some remarks. Note that, in this section, the term fan does not necessarily mean a rational fan.

First, we regard a moment-angle manifold as a submanifold of $\mathbb{C}^{m}$ in the following way. Suppose that a simple $n$-polytope $P$ with $m$ facets is realized in $\mathbb{R}^{n}$ by

$$
P=\left\{\mathbf{x} \in \mathbb{R}^{n} \mid\left\langle\mathbf{n}_{i}, \mathbf{x}\right\rangle+\gamma_{i} \geq 0 \quad(1 \leq i \leq m)\right\}
$$

where $\mathbf{n}_{i} \in \mathbb{R}^{n}, \gamma_{i} \in \mathbb{R}$ and $\langle$,$\rangle denotes the standard scalar product on \mathbb{R}^{n}$. Then we identify $\mathcal{Z}_{P}$ with the image of

$$
\left\{(\mathbf{x}, \boldsymbol{z}) \in P \times\left.\mathbb{C}^{m}|| z_{i}\right|^{2}=\left\langle\mathbf{n}_{i}, \mathbf{x}\right\rangle+\gamma_{i} \quad(1 \leq i \leq m)\right\}, \quad \boldsymbol{z}=\left(z_{1}, \ldots, z_{m}\right),
$$

under the projection to the second component. Note that this set does not depend on whether $\mathbf{x}$ runs over $P$ or $\mathbb{R}^{n}$. Since we can choose $n$ vectors out of $\mathbf{n}_{1}, \ldots, \mathbf{n}_{m}$ so that they are linearly independent, we can delete $\mathbf{x}$ from the above $m$ equations to obtain $f_{i}: \mathbb{C}^{m} \rightarrow \mathbb{R}(i=1, \ldots, m-n)$ such that $\mathcal{Z}_{P}=\bigcap_{i=1}^{m-n} f_{i}^{-1}(0)$.

Let $\mathcal{K}$ be the simplicial complex on $[m]$ whose geometric realization is $\partial P^{*}$, and put

$$
U(\mathcal{K})=\mathbb{C}^{m} \backslash \bigcup_{I \notin \mathcal{K}}\left\{\boldsymbol{z} \in \mathbb{C}^{m} \mid z_{i}=0(i \in I)\right\} .
$$

If we denote by $\lambda^{\mathbb{R}}:\left(\mathbb{R}_{>0}\right)^{m} \rightarrow\left(\mathbb{R}_{>0}\right)^{n}$ the homomorphism sending $\left(y_{1}, \ldots, y_{m}\right)$ to $y_{1}^{\mathbf{n}_{1}} \cdots y_{m}^{\mathbf{n}_{m}}$, where $y^{\mathbf{u}}=\left(y^{u_{1}}, \ldots, y^{u_{n}}\right) \in\left(\mathbb{R}_{>0}\right)^{n}$ for $y \in \mathbb{R}_{>0}$ and $\mathbf{u}=\left(u_{1}, \ldots, u_{n}\right) \in$ $\mathbb{R}^{n}$, then we have the following. 
Proposition A.1 (cf. [16, Theorem 3.3]). The group $\operatorname{ker} \lambda^{\mathbb{R}}$ acts on $U(\mathcal{K})$ freely and properly. Moreover, the inclusion $\mathcal{Z}_{P} \rightarrow \mathbb{C}^{m}$ induces an $\left(S^{1}\right)^{m}$-equivariant diffeomorphism $\mathcal{Z}_{P} \rightarrow U(\mathcal{K}) / \operatorname{ker} \lambda^{\mathbb{R}}$.

More generally, for any simplicial fan $\left(\mathcal{K},\left\{\mathbf{n}_{i}\right\}_{i=1}^{m}\right)$, ker $\lambda^{\mathbb{R}}$ acts on $U(\mathcal{K})$ freely and properly. The following lemma is due to Hiroaki Ishida.

Lemma A.2. Let $\mathbf{n}_{i}(t)(i=1, \ldots, m)$ be a smooth function $[0,1] \rightarrow \mathbb{R}^{n}$ and suppose that $\left(\mathcal{K},\left\{\mathbf{n}_{i}(t)\right\}_{i=1}^{m}\right)$ is a simplicial fan for each $t$ in $[0,1]$. Then $U(\mathcal{K}) / \operatorname{ker} \lambda^{\mathbb{R}}(0)$ is $\left(S^{1}\right)^{m}$-equivariantly diffeomorphic to $U(\mathcal{K}) / \operatorname{ker} \lambda^{\mathbb{R}}(1)$.

Proof. Let $Y$ be the quotient space $U(\mathcal{K}) \times[0,1] / \sim$ where the equivalence relation $\sim$ is defined so that $(\boldsymbol{z}, t) \sim\left(\boldsymbol{z}^{\prime}, t^{\prime}\right)$ if and only if $t=t^{\prime}$ and $\boldsymbol{z}, \boldsymbol{z}^{\prime}$ are in the same orbit of $\operatorname{ker} \lambda^{\mathbb{R}}(t)$. Then we can easily verify that the projection to the second component descends to an $\left(S^{1}\right)^{m}$-equivariant smooth fiber bundle $Y \rightarrow[0,1]$. Since $[0,1]$ is contractible, we obtain the lemma.

Let $\Delta=(\mathcal{K}, \mathcal{V})$ be a complete nonsingular fan (see Section 2). Then the homomorphism $\lambda_{\mathcal{V}}:\left(\mathbb{C}^{*}\right)^{m} \rightarrow\left(\mathbb{C}^{*}\right)^{n}$ was defined in the same way as $\lambda^{\mathbb{R}}$ above. We note that $\left(\mathbb{C}^{*}\right)^{m}=\left(\mathbb{R}_{>0}\right)^{m} \times\left(S^{1}\right)^{m}$ and denote by $\lambda_{\mathcal{V}}^{\mathbb{R}}$ and $\lambda_{\mathcal{V}}^{S}$ the restrictions of $\lambda_{\mathcal{V}}$ to $\left(\mathbb{R}_{>0}\right)^{m}$ and $\left(S^{1}\right)^{m}$ respectively. Then since

$$
X(\Delta)=U(\mathcal{K}) / \operatorname{ker} \lambda_{\mathcal{V}}=\left(U(\mathcal{K}) / \operatorname{ker} \lambda_{\mathcal{V}}^{\mathbb{R}}\right) / \operatorname{ker} \lambda_{\mathcal{V}}^{S},
$$

we have the following corollary from Propostion A.1 and Lemma A.2.

Corollary A.3. If there is a smooth deformation of simplicial fan between a complete nonsingular fan $\Delta=(\mathcal{K}, \mathcal{V})$ and the normal fan of a simple polytope $P$ which is not necessarily a rational fan, then the toric manifold $X(\Delta)$ associated with $\Delta$ is $\left(S^{1}\right)^{n}$-equivariantly diffeomorphic to $\mathcal{Z}_{P} / \operatorname{ker} \lambda_{\mathcal{V}}^{S}$.

Proof of Proposition 7.2. Consider the deformation given by

$$
A(t)=\left(\mathbf{a}_{1}(t), \ldots, \mathbf{a}_{n}(t)\right)=\left[\begin{array}{ccccc}
1 & 0 & \ldots & 0 & a_{1} t \\
a_{2} t & 1 & \ldots & 0 & 0 \\
\vdots & \vdots & \ddots & \vdots & \vdots \\
0 & 0 & \ldots & 1 & 0 \\
0 & 0 & \ldots & a_{n} t & 1
\end{array}\right], \quad \mathbf{b}(t)=\frac{1}{2} \sum_{i=1}^{n} \mathbf{a}_{i}(t) .
$$

We can easily verify that this is a smooth family of simplicial fans. Since the column vectors of $\left(-E_{n}, A(0), \mathbf{b}(0)\right)$ are the normal vectors of $P$ in (7.1), where $E_{n}$ denotes the identity matrix, the proposition follows from Corollary A.3.

We finish this paper with three remarks.

Remark 2. Every toric manifold of Type 2 is over vc $\left(I^{n}\right)$ from Proposition 7.2 Hence, together with Proposition 8.3, we can conclude that the toric manifold associated with a fan over $\mathcal{C}_{\mathrm{n}}$ is over $\operatorname{vc}\left(I^{n}\right)$.

Remark 3. Delaunay [9] proves that if there exists a projective toric manifold over a simple 3-dimensional polytope $P$, then $P$ has at least one triangular or quadrangular face (see also [1]). On the other hand, Suyama [19] shows that any triangulation of 2-sphere with at most 18 vertices can be the underlying simplicial complex of a complete nonsingular fan of dimension 3. Combining these two results, we can see that there exist many non-projective toric manifolds of complex dimension 3 . 
Remark 4. Hirzebruch surface $F_{a}$, where $a$ is a nonnegative integer, is the total space of the projective bundle $\mathbb{P}(\underline{\mathbb{C}} \oplus \mathcal{O}(a))$ over $\mathbb{P}^{1}$, where $\underline{\mathbb{C}}$ denotes the trivial line bundle over $\mathbb{P}^{1}$ and $\mathcal{O}(a)$ denotes the complex line bundle over $\mathbb{P}^{1}$ whose first Chern class is $a$ times a generator of $H^{2}\left(\mathbb{P}^{1}\right)$. Therefore, $F_{a}$ is a Bott manifold. As is well-known, $F_{a}$ 's are not isomorphic to each other as varieties but $F_{a}$ and $F_{b}$ are diffeomorphic if and only if $a \equiv b(\bmod 2)$. The latter fact can be proved in an elementary way but it can also be proved using deformations of complex structures as is well-known. So, looking at Propositions 6.1] and 7.1, it would be interesting to ask whether there exist deformations of complex structures on our toric manifolds of Type 0 or 2 . See [17] for related work.

\section{ACKNOWLEDGEMENTS}

The authors thank Suyoung Choi for providing the proof of Lemma 5.4 when $n=3$ and Hiroaki Ishida for his help on moment-angle manifolds.

\section{REFERENCES}

[1] A. Ayzenberg, Toric manifolds over 3-polytopes, arXiv:1607.03377

[2] V. M. Buchstaber and T. E. Panov, Toric Topology, Mathematical Surveys and Monographs, 204. American Mathematical Society, Providence, RI, 2015.

[3] S. Choi, Classification of Bott manifolds up to dimension eight, P. Edinburgh Math. Soc. (2) 58 (2015), 653-659.

[4] S. Choi and M. Masuda, Classification of $\mathbb{Q}$-trivial Bott manifolds, J. Symplectic Geom. 10 (2012), no. 3, 447-461.

[5] S. Choi, M. Masuda, and S. Murai, Invariance of Pontrjagin classes for Bott manifolds, Algebr. Geom. Topol. 15 (2015), no. 2, 965-986.

[6] S. Choi, M. Masuda, and D. Y. Suh, Topological classification of generalized Bott towers, Trans. Amer. Math. Soc. 362 (2010), 1097-1112.

[7] S. Choi, T. Panov, and D. Y. Suh, Toric cohomological rigidity of simple convex polytopes, J. London Math. Soc. 82 (2010), 343-360.

[8] D. A. Cox, J. B. Little, and H. K. Schenck, Toric Varieties, Graduate Studies in Mathematics, vol. 124, American Mathematical Society, Providence, RI, 2011.

[9] C. Delaunay, On hyperbolicity of toric real threefolds, IMRN 51 (2005), 3191-3201.

[10] W. Fulton, An Introduction to Toric Varieties, Ann. of Math. Studies, vol. 113, Princeton Univ. Press, Princeton, N.J., 1993.

[11] M. Grossberg and Y. Karshon, Bott towers, complete integrability, and the extended character of representations, Duke Math. J. 76 (1994), 23-58.

[12] M. Masuda and T. E. Panov, Semifree circle actions, Bott towers, and quasitoric manifolds, Sbornik Math. 199:8, (2008), 1201-1223,

[13] M. Masuda and D. Y. Suh, Classification problems of toric manifolds via topology, Proc. of Toric Topology, Contemp. Math. 460: 273-286, 2008.

[14] A. Mukherjee, Differential Topology, Second edition. Hindustan Book Agency, New Delhi; Birkhäuser/Springer, Cham, 2015.

[15] T. Oda, Convex Bodies and Algebraic Geometry. An Introduction to the Theory of Toric Varieties, Ergeb. Math. Grenzgeb. (3), 15, Springer-Verlag, Berlin, 1988.

[16] T. E. Panov and Y. Ustinovsky, Complex geometry of moment-angle manifolds, Moscow Math. J. 12 (2012), 149-172.

[17] H. Sato, Jumping deformations of complete toric varieties, Int. J. Math. Math. Sci. 49 (2003), 3101-3113.

[18] Y. Suyama, Examples of toric manifolds which are not quasitoric manifolds, Algebr. Geom. Topol. 14 (2014), 3097-3106.

[19] Y. Suyama, Simplicial 2-spheres obtained from non-singular complete fans, Del'nevost. Mat. Zh. 15, no. 2 (2015), 277-288.

[20] The on-line encyclopedia of integer sequences, available at https://oeis.org/. 
Faculty of Liberal Arts and Sciences, Osaka Prefecture University, Osaka 599-8531, JAPAN.

E-mail address: s.hasui@las.osakafu-u.ac.jp

Department of Mathematics, Osaka City University, Sumiyoshi-ku, Osaka 558-8585, JAPAN.

E-mail address: hideya0813@gmail.com

Department of Mathematics, Osaka City University, Sumiyoshi-Ku, Osaka 558-8585, JAPAN.

E-mail address: masuda@sci.osaka-cu.ac.jp

Department of Mathematics, Osaka City University, Sumiyoshi-Ku, Osaka 558-8585, JAPAN.

E-mail address: seonjeong1124@gmail.com 